\title{
Duality and biorthogonality for g-frames in Hilbert spaces
}

\author{
Farideh Enayati, Mohammad Sadegh Asgari* \\ Department of Mathematics, Faculty of Science, Central Tehran Branch, Islamic Azad University, Tehran, Iran.
}

\begin{abstract}
The main aim of this paper is to define the generalized Riesz-dual sequence from a g-Bessel sequence with respect to a pair of g-orthonormal bases. We characterize exactly properties of the first sequence in terms of the associated one, which yields duality relations for the abstract g-frame setting. (C)2017 all rights reserved.
\end{abstract}

Keywords: g-orthonormal basis, g-frames, g-Riesz-dual sequence, Riesz-duality. 2010 MSC: 41A58, 42A38, 42C15, 42C40.

\section{Introduction}

Duality principles in Gabor theory such as the Ron-Shen duality principle [13] and the Wexler-Raz biorthogonality relations [17] play a fundamental role for analyzing Gabor systems. Casazza et al. in [4] introduced a general approach to derive duality principles in abstract frame theory. For each sequence in a separable Hilbert space they defined a Riesz-dual sequence dependent only on two orthonormal bases. They characterize exactly properties of the first sequence in terms of the Riesz-dual sequence, which yields duality relations for the frame setting. Frames were first introduced by Duffin and Schaeffer [9] in the context of nonharmonic Fourier series and reintroduced in 1986 by Daubechies et al. in [8]. Currently, frames play important roles in many applications in mathematics, science, and engineering such as signal processing, image processing, data compression, etc.

Let $\left\{e_{i}\right\}_{i \in I}$ and $\left\{h_{i}\right\}_{i \in I}$ be orthonormal bases for a separable Hilbert space $\mathcal{H}$ and let $f=\left\{f_{i}\right\}_{i \in I}$ be any sequence in $\mathcal{H}$ for which $\sum_{i \in I}\left|\left\langle f_{i}, e_{j}\right\rangle\right|^{2}<\infty$ for all $j \in I$. Then the Riesz-dual sequence (R-dual sequence) of $\left\{f_{i}\right\}_{i \in I}$ with respect to $\left\{e_{i}\right\}_{i \in I}$ and $\left\{h_{i}\right\}_{i \in I}$ as the sequence $\left\{\mathscr{W}_{j}^{f}\right\}_{j \in I}$ is given by:

$$
\mathscr{W}_{j}^{\mathrm{f}}=\sum_{i \in \mathrm{I}}\left\langle\mathrm{f}_{\mathrm{i}}, e_{\mathrm{j}}\right\rangle \mathrm{h}_{\mathrm{i}}, \quad \forall \mathrm{j} \in \mathrm{I} .
$$

This simple construction gives a powerful tool for deriving duality principles in general frame theory. There exists a symmetric relation between the sequences $\left\{\mathscr{W}_{j}^{f}\right\}_{j \in I}$ and $\left\{f_{i}\right\}_{i \in I}$ as follows:

$$
f_{i}=\sum_{j \in I}\left\langle\mathscr{W}_{j}^{f}, h_{i}\right\rangle e_{j}, \quad \forall i \in I .
$$

\footnotetext{
*Corresponding author

Email address: faridehenayati372@yahoo.com (Farideh Enayati)
} 
In particular, this shows that $\left\{f_{i}\right\}_{i \in I}$ is the R-dual sequence for $\left\{\mathscr{W}_{j}^{f}\right\}_{j \in I}$ with respect to $\left\{h_{i}\right\}_{i \in I}$ and $\left\{e_{i}\right\}_{i \in I}$. We refer the reader to the articles $[6,7,14,18]$ for an introduction about the theory and applications of R-dual sequences.

Recently, Sun in $[15,16]$ and Casazza and Kutyniok in [3] introduced a generalization of frames which covers many other recent generalizations of frames, e.g., bounded quasi-projectors, frames of subspaces, outer frames, oblique frames, pseudo-frames, and a class of time-frequency localization operators. Sun showed that all of the above applications of frames are special cases of generalized frames.

Let $\mathcal{H}$ and $\mathcal{K}$ be two separable Hilbert spaces and let $\left\{V_{i}\right\}_{i \in I}$ be a family of closed subspaces of $\mathcal{K}$ and $B\left(\mathcal{H}, V_{i}\right)$ denote the collection of all bounded linear operators from $\mathcal{H}$ into $V_{i}$ for all $i \in I$. Then, $\Lambda=\left\{\Lambda_{i} \in B\left(\mathcal{H}, V_{i}\right): i \in I\right\}$ is a generalized frame or simply a g-frame for $\mathcal{H}$ with respect to $\left\{V_{i}\right\}_{i \in I}$ if there exist constants $0<\mathrm{C} \leqslant \mathrm{D}<\infty$ such that:

$$
C\|f\|^{2} \leqslant \sum_{i \in I}\left\|\Lambda_{i} f\right\|^{2} \leqslant D\|f\|^{2}, \quad \forall f \in \mathcal{H}
$$

The constants C and D are called g-frame bounds. If only the right-hand inequality of (1.1) is required, we call it a g-Bessel sequence. Since almost all applications require a finite model for their numerical treatment, we restrict ourselves to a finite-dimensional space in the following examples.

Example 1.1. Let $\mathcal{H}=\mathbb{C}^{n}$ and $V_{1}=V_{2}=\ldots=V_{n}=\mathbb{C}^{n+1}$. Define

$$
\Lambda_{1}=\left[\begin{array}{cccc}
1 & 0 & \ldots & 0 \\
1 & 0 & \ldots & 0 \\
0 & 0 & \ldots & 0 \\
\vdots & \vdots & & \vdots \\
0 & 0 & \ldots & 0
\end{array}\right], \Lambda_{2}=\left[\begin{array}{cccc}
0 & 1 & \ldots & 0 \\
0 & 1 & \ldots & 0 \\
0 & 1 & \ldots & 0 \\
\vdots & \vdots & & \vdots \\
0 & 0 & \ldots & 0
\end{array}\right], \ldots, \wedge_{n}=\left[\begin{array}{cccc}
0 & 0 & \ldots & 1 \\
0 & 0 & \ldots & 1 \\
0 & 0 & \ldots & 1 \\
\vdots & \vdots & & \vdots \\
0 & 0 & \ldots & 1
\end{array}\right]
$$

Then, the set $\Lambda=\left\{\Lambda_{i}\right\}_{i=1}^{n}$ is a $g$-frame for $\mathbb{C}^{n}$ with respect to $\mathbb{C}^{n+1}$ with $g$-frame bounds $A=2$ and $\mathrm{B}=\mathrm{n}+1$. To see this explicitly, note that for any $\mathrm{f}=\left(z_{1}, z_{2}, \ldots, z_{\mathfrak{n}}\right)$ in $\mathbb{C}^{\mathfrak{n}}$, we have

$$
\sum_{i=1}^{n}\left\|\Lambda_{i} f\right\|^{2}=2\left|z_{1}\right|^{2}+3\left|z_{2}\right|^{2}+\ldots+(n+1)\left|z_{n}\right|^{2} .
$$

From this, we have

$$
2\|f\|^{2} \leqslant \sum_{i=1}^{n}\left\|\Lambda_{i} f\right\|^{2} \leqslant(n+1)\|f\|^{2} .
$$

In frames theory an input signal is represented by a collection of scalar coefficients that measure the projection of that signal onto each frame vector. The representation space employed in this theory equals $\ell^{2}(\mathrm{I})$. However, in g-frames theory an input signal is represented by a collection of vector coefficients that represent the projection (not just the projection energy) onto each subspace. Therefore the representation space employed in this setting is

$$
\left(\sum_{i \in I} \oplus V_{i}\right)_{\ell^{2}}=\left\{\left\{g_{i}^{\prime}\right\}_{i \in I} \mid g_{i}^{\prime} \in V_{i}, \sum_{i \in I}\left\|g_{i}^{\prime}\right\|^{2}<\infty\right\}
$$

In order to analyze a signal $f \in \mathcal{H}$, i.e., to map it into the representation space, the analysis operator $\mathrm{T}_{\Lambda}$ : $\mathcal{H} \rightarrow\left(\sum_{i \in I} \oplus V_{i}\right)_{\ell^{2}}$ given by $T_{\Lambda} f=\left\{\Lambda_{i} f\right\}_{i \in I}$ is applied. The associated synthesis operator, which provides a mapping from the representation space to $\mathcal{H}$, is defined to be the adjoint operator $\mathrm{T}_{\Lambda}^{*}:\left(\sum_{i \in \mathrm{I}} \oplus \mathrm{V}_{\mathrm{i}}\right)_{\ell^{2}} \rightarrow$ $\mathcal{H}$, which is given by $\mathrm{T}_{\Lambda}^{*}\left(\left\{g_{i}^{\prime}\right\}_{i \in \mathrm{I}}\right)=\sum_{i \in \mathrm{I}} \Lambda_{i}^{*} g_{i}^{\prime}$. By composing $\mathrm{T}_{\Lambda}$ and $\mathrm{T}_{\Lambda}^{*}$ we obtain the g-frame operator $S_{\Lambda}: \mathcal{H} \rightarrow \mathcal{H}, S_{\Lambda} f=T_{\Lambda}^{*} T_{\Lambda} f=\sum_{i \in I} \Lambda_{i}^{*} \Lambda_{i} f$, which is a positive, self-adjoint and invertible operator and 
$C \leqslant\left\|S_{\Lambda}\right\| \leqslant D$. The canonical dual g-frame for $\left\{\Lambda_{i}\right\}_{i \in I}$ is defined by $\left\{\widehat{\Lambda}_{i}\right\}_{i \in I}$ where $\widehat{\Lambda}_{i}=\Lambda_{i} S_{\Lambda}^{-1}$ which is also a g-frame for $\mathcal{H}$ with respect to $\left\{\mathrm{V}_{i}\right\}_{i \in \mathrm{I}}$ with $\frac{1}{\mathrm{D}}$ and $\frac{1}{\mathrm{C}}$ as its lower and upper frame bounds, respectively. Also we have

$$
f=\sum_{i \in I} \Lambda_{i}^{*} \widehat{\Lambda}_{i} f=\sum_{i \in I} \widehat{\Lambda}_{i}^{*} \Lambda_{i} f, \quad \forall f \in \mathcal{H} .
$$

Moreover, $\left\{\Lambda_{i} S_{\Lambda}^{-\frac{1}{2}}\right\}_{i \in I}$ is a Parseval g-frame for $\mathcal{H}$ with respect to $\left\{\bigvee_{i}\right\}_{i \in I}$.

Generalized Riesz-dual sequence or simply g-R-dual sequence is a natural generalization of R-dual sequence which provides a powerful tool in the analysis of duality relations in general g-frame theory. The purpose of this paper is to introduce the concept of Riesz-dual sequence for g-frames. We give characterizations of g-R-dual sequences and prove that g-R-dual sequences share many useful properties with R-dual sequences. In this article, we show that in fact for each sequence of operators we can construct a corresponding sequence of operators with a kind of duality relation between them. This construction is used to prove duality principles in g-frame theory, which can be regarded as general versions of several well-known duality principles for g-frames. We also give a generalized version of Riesz-dual sequences.

The content of this paper is as follows: In the rest of this section we will briefly recall the necessary parts from g-bases, g-orthonormal bases, and g-Riesz bases. For more information we refer to [1, 2, 5, 10, 11]. In Section 2, we define the g-R-dual sequence from a g-Bessel sequence with respect to a pair of g-orthonormal bases as generalization of Riesz-dual sequence. In this section, we characterize to which extent the g-R-dual sequence of a g-Bessel sequence depends on the chosen g-orthonormal bases. In Section 3, first we obtain the g-frame conditions for a sequence of operators and its g-R-dual sequence. We also characterize those pairs of g-frames and their g-R-dual sequences, which are equivalent (unitarily equivalent). Finally, Section 4 deals with duality principle for g-frames. In this section we study properties of dual g-frames and canonical dual g-frames.

Definition 1.2. A generalized Schauder basis or simply a g-basis for $\mathcal{H}$ with respect to $\left\{W_{i}\right\}_{i \in I}$ is a family of onto operators $\Gamma=\left\{\Gamma_{j} \in \mathrm{B}\left(\mathcal{H}, W_{j}\right) \mid j \in I\right\}$ such that for all $f \in \mathcal{H}$ there exist unique vectors $g_{j} \in W_{j}, i \in I$ with

$$
f=\sum_{j \in I} \Gamma_{j}^{*} g_{j} .
$$

In this case, there exist unique operators $\Lambda_{j} \in B\left(\mathcal{H}, W_{j}\right)$ such that

$$
f=\sum_{j \in I} \Gamma_{j}^{*} \Lambda_{j} f=\sum_{j \in I} \Lambda_{j}^{*} \Gamma_{j} f,
$$

for all $f \in \mathcal{H}$. Moreover, the sequences $\left\{\Gamma_{j}\right\}_{j \in I}$ and $\left\{\Lambda_{j}\right\}_{j \in I}$ are g-biorthogonal, i.e., $\Lambda_{i} \Gamma_{j}^{*} g_{j}=\delta_{i j} g_{j}$ for all $i, j \in I, g_{j} \in W_{j}$ and $\left\{\Lambda_{j}\right\}_{j \in I}$ itself forms a g-basis for $\mathcal{H}$ with respect to $\left\{W_{i}\right\}_{i \in I}$ that so-called dual g-basis of $\left\{\Gamma_{j}\right\}_{j \in I}$. A g-basis is an unconditional g-basis, if the series in (1.2) converges unconditionally. Consequently, for a g-basis the ordering in (1.2) can be crucial. If $\left\{\Lambda_{i}\right\}_{i \in I}$ is a g-basis only for its closed linear span, we call it a g-basic sequence with respect to $\left\{W_{i}\right\}_{i \in I}$.

Definition 1.3. Let $\left\{\Xi_{i} \in B\left(\mathcal{H}, W_{i}\right) \mid i \in I\right\}$ be a sequence of operators. Then

(i) $\left\{\Xi_{i}\right\}_{i \in I}$ is a g-complete set for $\mathcal{H}$ with respect to $\left\{W_{i}\right\}_{i \in I}$, if $\mathcal{H}=\overline{\operatorname{span}}\left\{\Xi_{i}^{*}\left(W_{i}\right)\right\}_{i \in I}$.

(ii) $\left\{\Xi_{i}\right\}_{i \in I}$ is a g-orthonormal system for $\mathcal{H}$ with respect to $\left\{W_{i}\right\}_{i \in I}$, if $\Xi_{i} \Xi_{j}^{*}=\delta_{i j} I_{W_{j}}$ for all $i, j \in I$.

(iii) A g-complete and g-orthonormal system $\left\{\Xi_{i}\right\}_{i \in I}$ is called a g-orthonormal basis for $\mathcal{H}$ with respect to $\left\{W_{i}\right\}_{i \in I}$.

Definition 1.4. A sequence $\Gamma=\left\{\Gamma_{\mathbf{j}} \in \mathrm{B}\left(\mathcal{H}, W_{\mathbf{j}}\right) \mid j \in I\right\}$ is called a g-Riesz basis for $\mathcal{H}$ with respect to $\left\{W_{\mathbf{j}}\right\}_{j \in I}$, if $\left\{\Gamma_{j}\right\}_{j \in I}$ is a g-complete set for $\mathcal{H}$ with respect to $\left\{W_{j}\right\}_{j \in I}$ and there exist constants $0<A \leqslant B<\infty$ such that

$$
A \sum_{j \in I}\left\|g_{j}\right\|^{2} \leqslant\left\|\sum_{j \in I} \Gamma_{j}^{*} g_{j}\right\|^{2} \leqslant B \sum_{j \in I}\left\|g_{j}\right\|^{2},
$$


for all sequences $\left\{g_{j}\right\}_{j \in I} \in\left(\sum_{j \in I} \oplus W_{j}\right)_{\ell^{2}}$. We define the g-Riesz basis bounds for $\left\{\Gamma_{j}\right\}_{j \in I}$ to be the largest number $A$ and the smallest number $B$ such that this inequality (1.3) holds. If $\left\{\Gamma_{j}\right\}_{j \in I}$ is a g-Riesz basis only for $\overline{\operatorname{span}}\left\{\Gamma_{j}^{*}\left(W_{j}\right)\right\}_{j \in I}$, we call it a g-Riesz basic sequence for $\mathcal{H}$ with respect to $\left\{W_{j}\right\}_{j \in I}$.

The following well-known characterization of g-orthonormal bases is sometimes more useful which is taken from [2].

Lemma 1.5. Let $\Xi=\left\{\Xi_{i}\right\}_{i \in \mathrm{I}}$ be a g-orthonormal system for $\mathcal{H}$ with respect to $\left\{\mathrm{W}_{\boldsymbol{i}}\right\}_{\mathbf{i} \in \mathrm{I}}$. Then the following conditions are equivalent:

(i) $\Xi$ is a g-orthonormal basis for $\mathcal{H}$ with respect to $\left\{\mathrm{W}_{i}\right\}_{i \in \mathrm{I}}$.

(ii) $\sum_{i \in I} \Xi_{i}^{*} \Xi_{i}=I_{\mathcal{H}}$.

(iii) $\|\mathrm{f}\|^{2}=\sum_{i \in \mathrm{I}}\left\|\Xi_{i}^{*} \Xi_{i} f\right\|^{2}, \quad \forall \mathrm{f} \in \mathcal{H}$.

(iv) $\|\mathrm{f}\|^{2}=\sum_{i \in \mathrm{I}}\left\|\Xi_{i} f\right\|^{2}, \quad \forall \mathrm{f} \in \mathcal{H}$.

(v) $<f, g>=\sum_{i \in I}<\Xi_{i} f, \Xi_{i} g>, \quad \forall f, g \in \mathcal{H}$.

(vi) If $\Xi_{i} f=0$ for all $i \in I$, then $f=0$.

For any given g-frame there is a natural procedure to construct a g-Riesz basis with the same g-frame bounds, see, e.g., [1] for a proof of this standard result.

Lemma 1.6. Let $\left\{\Xi_{\mathbf{j}}\right\}_{j \in \mathrm{I}}$ be a g-orthonormal system for $\mathcal{H}$ with respect to $\left\{\mathrm{W}_{\mathbf{j}}\right\}_{j \in \mathrm{I}}$ and $\mathrm{U}: \mathcal{H} \rightarrow \mathcal{H}$ a bounded bijective operator. Then the following items hold.

(i) The sequence $\left\{\Xi_{j} \mathrm{U}^{*}\right\}_{j \in \mathrm{I}}$ is a g-Riesz basis for $\mathcal{H}$ with respect to $\left\{\mathrm{W}_{\mathbf{j}}\right\}_{j \in \mathrm{I}}$ with g-frame operator $\mathrm{UU}^{*}$ and optimal bounds $\frac{1}{\left\|\mathrm{U}^{-1}\right\|^{2}},\|\mathrm{U}\|^{2}$.

(ii) The dual g-Riesz basis of $\left\{\Xi_{\mathbf{j}} \mathrm{U}^{*}\right\}_{\mathbf{j} \in \mathrm{I}}$ is $\left\{\Xi_{\mathbf{j}} \mathrm{U}^{-1}\right\}_{\mathbf{j} \in \mathrm{I}}$ with $g$-frame operator ( $\left.\mathrm{UU}^{*}\right)^{-1}$ and the optimal bounds are $\frac{1}{\|\mathrm{u}\|^{2}},\left\|\mathrm{U}^{-1}\right\|^{2}$.

(iii) Let $\Gamma=\left\{\Gamma_{\mathbf{j}}\right\}_{j \in \mathrm{I}}$ be a g-frame for $\mathcal{H}$ with respect to $\left\{\mathrm{W}_{\mathbf{j}}\right\}_{\mathbf{j} \in \mathrm{I}}$ with optimal bounds $\mathrm{A}$, B. Then $\left\{\Xi_{\mathrm{j}} S_{\Gamma}^{\frac{1}{2}}\right\}_{j \in \mathrm{I}}$ is a g-Riesz basis for $\mathcal{H}$ with respect to $\left\{W_{\mathbf{j}}\right\}_{j \in \mathrm{I}}$ with optimal bounds $A$, B. The dual g-Riesz basis of $\left\{\Xi_{\mathbf{j}} \mathrm{S}_{\Gamma}^{\frac{1}{2}}\right\}_{\mathbf{j} \in \mathrm{I}}$ is $\left\{\Xi_{\mathfrak{j}} S_{\Gamma}^{-\frac{1}{2}}\right\}_{j \in \mathrm{I}}$, with optimal bounds $\frac{1}{\mathrm{~B}}, \frac{1}{\mathrm{~A}}$.

(iv) Let $\Gamma=\left\{\Gamma_{j}\right\}_{j \in \mathrm{I}}$ be a g-Riesz basis for $\mathcal{H}$ with respect to $\left\{\mathrm{W}_{\mathbf{j}}\right\}_{j \in \mathrm{I}}$, then $\left\{\Gamma_{\mathrm{j}} \mathrm{S}_{\Gamma}^{-\frac{1}{2}}\right\}_{\mathbf{j} \in \mathrm{I}}$ is a g-orthonormal basis for $\mathcal{H}$ with respect to $\left\{W_{j}\right\}_{j \in I}$.

(v) Let $\Gamma=\left\{\Gamma_{j} \in \mathrm{B}\left(\mathcal{H}, W_{j}\right) \mid j \in I\right\}$ be arbitrary sequence. If $\overline{\operatorname{span}}\left\{\Gamma_{j}^{*}\left(W_{j}\right)\right\}_{j \in I}=\mathcal{H}$ and

$$
\left\|\sum_{j \in I} \Gamma_{j}^{*} g_{j}\right\|^{2}=\sum_{j \in I}\left\|g_{j}\right\|^{2}, \quad \forall\left\{g_{j}\right\}_{j \in I} \in\left(\sum_{j \in I} \oplus W_{j}\right)_{\ell^{2}}
$$

then $\Gamma=\left\{\Gamma_{\mathbf{j}}\right\}_{\mathbf{j} \in \mathrm{I}}$ is a g-orthonormal basis for $\mathcal{H}$ with respect to $\left\{\mathrm{W}_{\mathrm{i}}\right\}_{\mathbf{i} \in \mathrm{I}}$.

Let $\Xi=\left\{\Xi_{i}\right\}_{i \in I}$ be a g-orthonormal basis for $\mathcal{H}$ with respect to $\left\{W_{i}\right\}_{i \in I}$. If $f=\sum_{i \in I} \Xi_{i}^{*} g_{i}$, then the coordinate representation of $f \in \mathcal{H}$ relative to the g-orthonormal basis $\Xi$ is $[\mathrm{f}]_{\Xi}=\left\{\mathrm{g}_{i}\right\}_{i \in \mathrm{I}}$. In this case $\left\{g_{i}\right\}_{i \in I} \in\left(\sum_{i \in I} \oplus W_{i}\right)_{\ell^{2}}$ and $\|f\|=\left\|[f]_{\Xi}\right\|_{\ell^{2}}$.

Definition 1.7. Let $\Xi=\left\{\Xi_{i}\right\}_{i \in I}$ and $\Xi^{\prime}=\left\{\Xi_{i}^{\prime}\right\}_{i \in I}$ be g-orthonormal bases for $\mathcal{H}$ with respect to $\left\{W_{i}\right\}_{i \in I}$ and $\left\{V_{i}\right\}_{i \in I}$, respectively. The transition matrix from $\Xi$ to $\Xi^{\prime}$ is the matrix $B=\left[B_{i j}\right]$ whose $(i, j)$-entry is $B_{i j}=\Xi_{i}^{\prime} \Xi_{j}^{*}$ for all $i, j \in I$. We also have $B[f]_{\Xi}=[f]_{\Xi^{\prime}}$ where, $[f]_{\Xi}$ and $[f]_{\Xi^{\prime}}$ are the coordinate representation of an arbitrary vector $f \in \mathcal{H}$ in the basis $\Xi$ and $\Xi^{\prime}$, respectively. We show that the transition matrix from $\Xi^{\prime}$ to $\Xi$ is $B^{-1}=B^{*}$. Let $B^{*}=\left[B_{i j}^{*}\right]$, then $B_{i j}^{*}=\left(B_{j i}\right)^{*}=\Xi_{i} \Xi_{j}^{\prime *}$ for all $i, j \in I$. By Lemma 1.5 we have

$$
\left[B B^{*}\right]_{i j}=\sum_{k \in I} B_{i k} B_{k j}^{*}=\sum_{k \in I} E_{i}^{\prime} E_{k}^{*} E_{k} E_{j}^{\prime *}=E_{i}^{\prime}\left(\sum_{k \in I} E_{k}^{*} E_{k}\right) E_{j}^{\prime *}=E^{\prime}{ }_{i} I_{\mathcal{H}} E_{j}^{\prime *}=E^{\prime}{ }_{i} E_{j}^{\prime *}=\delta_{i j} I_{W_{j}} .
$$

Similarly, $\left[\mathrm{B}^{*} \mathrm{~B}\right]_{i j}=\delta_{i j} \mathrm{I}_{W_{j}}$. This implies that $\mathrm{BB}^{*}=\mathrm{B}^{*} \mathrm{~B}=\mathrm{I}$, where $\mathrm{I}$ is the identity matrix.

Since almost all applications require a finite model for their numerical treatment, we restrict ourselves to a finite-dimensional space in the following example. 
Example 1.8. Let $\mathcal{H}=\mathbb{C}^{2 n}$ and $W_{1}=W_{2}=\ldots=W_{n}=\mathbb{C}^{2}$. Define

$$
\Xi_{1}=\left[\begin{array}{lllll}
1 & 0 & \ldots & 0 & 0 \\
0 & 1 & \ldots & 0 & 0
\end{array}\right], \ldots, \Xi_{n}=\left[\begin{array}{ccccc}
0 & 0 & \ldots & 1 & 0 \\
0 & 0 & \ldots & 0 & 1
\end{array}\right] \text {. }
$$

A direct calculation shows that $\left\|\Xi_{k}\right\|=1$ and $\Xi_{k} \Xi_{\ell}^{*}=\delta_{k \ell}$ for any $1 \leqslant k, \ell \leqslant n$. We also have

$$
\sum_{k=1}^{n}\left\|\Xi_{k} f\right\|^{2}=\sum_{k=1}^{n}\left(\left|z_{2 k-1}\right|^{2}+\left|z_{2 k}\right|^{2}\right)=\|f\|^{2}, \quad \forall f=\left\{z_{i}\right\}_{i=1}^{2 n} \in \mathbb{C}^{2 n} .
$$

Therefore $\Xi=\left\{\Xi_{k}\right\}_{k=1}^{n}$ is a g-orthonormal basis for $\mathbb{C}^{2 n}$ with respect to $\mathbb{C}^{2}$. Similarly, the sequence $\Psi=\left\{\Psi_{k}\right\}_{k=1}^{n}$ defined by

$$
\Psi_{1}=\left[\begin{array}{lllll}
0 & 1 & \ldots & 0 & 0 \\
1 & 0 & \ldots & 0 & 0
\end{array}\right], \ldots, \Psi_{n}=\left[\begin{array}{lllll}
0 & 0 & \ldots & 0 & 1 \\
0 & 0 & \ldots & 1 & 0
\end{array}\right]
$$

is also a g-orthonormal basis for $\mathbb{C}^{2 n}$ with respect to $\mathbb{C}^{2}$ and the matrix

$$
B=\left[\Psi_{i} \Xi_{j}^{*}\right]_{n \times n}=\left[\begin{array}{ccc}
A & & \overline{0} \\
& \ddots & \\
\overline{0} & & A
\end{array}\right],
$$

where $A=\left[\begin{array}{ll}0 & 1 \\ 1 & 0\end{array}\right]$ is the transition matrix from $\Xi$ to $\Psi$. Hence, for any $f \in \mathbb{C}^{2 n}$ we have $B[f]_{\Xi}=[f]_{\Psi}$.

Example 1.9. Let $\mathcal{H}=\mathbb{C}^{2 n}$ and $W_{1}=W_{2}=\ldots=W_{2 n}=\mathbb{C}^{2}$. Define

$$
\Gamma_{1}=\left[\begin{array}{lllll}
1 & 0 & \ldots & 0 & 0 \\
0 & 2 & \ldots & 0 & 0
\end{array}\right], \ldots, \Gamma_{\mathrm{n}}=\left[\begin{array}{ccccc}
0 & 0 & \ldots & 2 \mathrm{n}-1 & 0 \\
0 & 0 & \ldots & 0 & 2 \mathrm{n}
\end{array}\right] .
$$

Since, for every $g_{i}=\left(z_{2 i-1}, z_{2 i}\right) \in \mathbb{C}^{2}$, we have $\left\|\sum_{i=1}^{n} \Gamma_{i}^{*} g_{i}\right\|^{2}=\sum_{i=1}^{2 n} i^{2}\left|z_{i}\right|^{2}$. Thus $\left\{\Gamma_{i}\right\}_{i=1}^{n}$ is a g-Riesz basis for $\mathbb{C}^{2 n}$ with respect to $\mathbb{C}^{2}$ with $g$-Riesz bounds 1 and $4 n^{2}$. Moreover, we can write $\left\{\Gamma_{i}\right\}_{i=1}^{n}=\left\{\Xi_{i} U^{*}\right\}_{i=1}^{n}$, where $\mathrm{U}$ is a bounded bijective operator defined by

$$
\mathrm{U}=\left[\begin{array}{cccc}
1 & 0 & \ldots & 0 \\
0 & 2 & \ldots & 0 \\
\vdots & \vdots & & \vdots \\
0 & 0 & \ldots & 2 \mathrm{n}
\end{array}\right]
$$

and $\Xi=\left\{\Xi_{k}\right\}_{k=1}^{n}$ is the g-orthonormal basis defined in Example 1.8.

\section{The g-R-dual sequence}

In this section we define the g-R-dual sequence from a sequence of operators. Then we exactly characterize to which extent the g-R-dual sequence of a g-Bessel sequence depends on the chosen g-orthonormal bases.

Definition 2.1. Let $\Xi=\left\{\Xi_{i}\right\}_{i \in I}$ and $\Psi=\left\{\Psi_{i}\right\}_{i \in I}$ be g-orthonormal bases for $\mathcal{H}$ with respect to $\left\{W_{i}\right\}_{i \in I}$ and $\left\{V_{i}\right\}_{i \in I}$, respectively. Let $\Lambda=\left\{\Lambda_{i}: \mathcal{H} \rightarrow V_{i} \mid i \in I\right\}$ be such that the series $\sum_{i \in I} \Lambda_{i}^{*} g_{i}^{\prime}$ is convergent for all $\left\{g_{i}^{\prime}\right\}_{i \in I} \in\left(\sum_{i \in I} \oplus V_{i}\right)_{\ell^{2}}$. For all $j \in I$, let

$$
\Gamma_{j}^{\wedge}: \mathcal{H} \rightarrow W_{j}, \quad \Gamma_{j}^{\wedge}=\sum_{i \in I} \Xi_{j} \Lambda_{i}^{*} \Psi_{i} .
$$

Then $\left\{\Gamma_{j}^{\wedge}\right\}_{j \in I}$ is called the generalized Riesz-dual sequence ( $g$-R-dual sequence) for the sequence $\Lambda$ with respect to $(\Xi, \Psi)$. 
Notice that the hypothesis that the series $\sum_{i \in I} \Lambda_{i}^{*} g_{i}^{\prime}$ is convergent for all $\left\{g_{i}^{\prime}\right\}_{i \in I} \in\left(\sum_{i \in I} \oplus V_{i}\right)_{\ell^{2}}$ is always fulfilled if the sequence $\Lambda=\left\{\Lambda_{i}\right\}_{i \in I}$ is g-Bessel sequence with respect to $\left\{V_{i}\right\}_{i \in I}$.

Example 2.2. Let $\mathcal{H}=\mathbb{C}^{2 n}$ and let $\left\{\Xi_{i}\right\}_{i=1}^{n},\left\{\Psi_{i}\right\}_{i=1}^{n}$ be the g-orthonormal bases for $\mathcal{H}$ with respect to $\mathbb{C}^{2}$ defined in Example 1.8. Define

$$
\Lambda_{1}=\left[\begin{array}{lllll}
1 & 1 & \ldots & 0 & 0 \\
0 & 1 & \ldots & 0 & 0
\end{array}\right], \ldots, \Lambda_{n}=\left[\begin{array}{lllll}
0 & 0 & \ldots & 1 & 1 \\
0 & 0 & \ldots & 0 & 1
\end{array}\right]
$$

Then, $\Lambda=\left\{\Lambda_{i}\right\}_{i=1}^{n}$ is a g-Bessel sequence for $\mathcal{H}$ with respect to $\mathbb{C}^{2}$ with g-Bessel bound $B=3$. The g-R-dual sequence for the sequence $\Lambda$ with respect to $(\Xi, \Psi)$ is defined as follows:

$$
\Gamma_{1}^{\wedge}=\left[\begin{array}{lllll}
0 & 1 & \ldots & 0 & 0 \\
1 & 1 & \ldots & 0 & 0
\end{array}\right], \ldots, \Gamma_{n}^{\wedge}=\left[\begin{array}{lllll}
0 & 0 & \ldots & 0 & 1 \\
0 & 0 & \ldots & 1 & 1
\end{array}\right]
$$

which is also a g-Bessel sequence for $\mathcal{H}$ with respect to $\mathbb{C}^{2}$ with g-Bessel bound $\mathrm{B}=3$.

Now, we need an algorithm to invert the process and calculate $\left\{\Lambda_{i}\right\}_{i \in I}$ from the sequence $\left\{\Gamma_{j}^{\wedge}\right\}_{j \in I}$.

Theorem 2.3. Let $\Xi=\left\{\Xi_{i}\right\}_{i \in I}$ and $\Psi=\left\{\Psi_{i}\right\}_{i \in I}$ be g-orthonormal bases for $\mathcal{H}$ with respect to $\left\{W_{i}\right\}_{i \in I}$ and $\left\{V_{i}\right\}_{i \in I}$, respectively. Let $\left\{\Lambda_{i}\right\}_{i \in I}$ be a g-Bessel sequence for $\mathcal{H}$ with respect to $\left\{\mathrm{V}_{\mathrm{i}}\right\}_{\mathrm{i} \in \mathrm{I}}$. Then, for all $\mathrm{i} \in \mathrm{I}$,

$$
\Lambda_{i}=\sum_{j \in I} \Psi_{i}\left(\Gamma_{j}^{\wedge}\right)^{*} \Xi_{j}
$$

In particular, this shows that $\left\{\Lambda_{i}\right\}_{i \in \mathrm{I}}$ is the $g-R$-dual sequence for $\left\{\Gamma_{j}^{\wedge}\right\}_{j \in \mathrm{I}}$ with respect to $(\Psi, \Xi)$.

Proof. The definition of $\left\{\Gamma_{j}^{\wedge}\right\}_{j \in I}$ implies that for every $i, j \in I$

$$
\Psi_{i}\left(\Gamma_{j}^{\wedge}\right)^{*}=\Psi_{i}\left(\sum_{k \in I} \Xi_{j} \Lambda_{k}^{*} \Psi_{k}\right)^{*}=\sum_{k \in I} \Psi_{i} \Psi_{k}^{*} \Lambda_{k} \Xi_{j}^{*}=\sum_{k \in I} \delta_{i k} \Lambda_{k} \Xi_{j}^{*}=\Lambda_{i} \Xi_{j}^{*} .
$$

Therefore $\Psi_{i}\left(\Gamma_{j}^{\wedge}\right)^{*}=\Lambda_{i} \Xi_{j}^{*}$. Now, by Lemma 1.5 we have

$$
\Lambda_{i}=\Lambda_{i} I_{\mathcal{H}}=\Lambda_{i}\left(\sum_{j \in I} \Xi_{j}^{*} \Xi_{j}\right)=\sum_{j \in I} \Lambda_{i} \Xi_{j}^{*} \Xi_{j}=\sum_{j \in I} \Psi_{i}\left(\Gamma_{j}^{\wedge}\right)^{*} \Xi_{j} .
$$

Definition 2.4. Let $\Xi=\left\{\Xi_{j}\right\}_{j \in I}$ be a g-orthonormal basis for $\mathcal{H}$ with respect to $\left\{W_{j}\right\}_{j \in I}$ and let $\Lambda=\left\{\Lambda_{i}\right\}_{i \in I}$ be a g-Bessel sequence for $\mathcal{H}$ with respect to $\left\{V_{i}\right\}_{i \in I}$ with the g-frame operator $S_{\Lambda}: \mathcal{H} \rightarrow \mathcal{H}$, respectively. Then the matrix representation of $S_{\Lambda}$ with respect to $\Xi$ is the matrix $\left[S_{\Lambda}\right]=\left[S_{i j}\right]$, with $S_{i j}=\Xi_{i} S_{\Lambda} \Xi_{j}^{*}$. Therefore

$$
\left[S_{\Lambda}\right]:\left(\sum_{i \in I} \oplus W_{i}\right)_{\ell^{2}} \rightarrow\left(\sum_{i \in I} \oplus W_{i}\right)_{\ell^{2}}, \quad \text { with } \quad\left[S_{\Lambda} f\right]_{\Xi}=\left[S_{\Lambda}\right][f]_{\Xi}, \quad \forall f \in \mathcal{H}
$$

Suppose $A=\left[A_{i j}\right]$ with $A_{i j}=\Lambda_{i} \Xi_{j}^{*}$, then $A^{*}=\left[A_{i j}^{*}\right]$ and $A_{i j}^{*}=\Xi_{i} \Lambda_{j}^{*}$ for all $i, j \in I$. Therefore

$$
A:\left(\sum_{i \in I} \oplus W_{i}\right)_{\ell^{2}} \rightarrow\left(\sum_{i \in I} \oplus V_{i}\right)_{\ell^{2}}, \quad \text { and } \quad A^{*} A:\left(\sum_{i \in I} \oplus W_{i}\right)_{\ell^{2}} \rightarrow\left(\sum_{i \in I} \oplus W_{i}\right)_{\ell^{2}}
$$

The matrix $A$ is called the analysis matrix for $\Lambda$ with respect to $\Xi$. A direct calculation shows that for every $f \in \mathcal{H}$ we have $A[f]_{\Xi}=T_{\wedge} f$. We also have

$$
\left[A^{*} A\right]_{i j}=\sum_{k \in I}\left[A^{*}\right]_{i k}[A]_{k j}=\sum_{k \in I} \Xi_{i} \Lambda_{k}^{*} \Lambda_{k} \Xi_{j}^{*}=\Xi_{i}\left(\sum_{k \in I} \Lambda_{k}^{*} \Lambda_{k}\right) \Xi_{j}^{*}=\Xi_{i} S_{\Lambda} \Xi_{j}^{*}=S_{i j}=\left[S_{\Lambda}\right]_{i j} .
$$

Thus, $A^{*} A=S_{\Lambda}$, where $A^{*} A=S_{\wedge}$ means that $A^{*} A=\left[S_{\Lambda}\right]$. 
The following result is a generalization of [4, Proposition 3] to g-frames about dependence of the g-R-dual sequence $\left\{\Gamma_{j}^{\wedge}\right\}_{j \in J}$ to choose the g-orthonormal bases $\Xi=\left\{\Xi_{i}\right\}_{i \in I}$ and $\Psi=\left\{\Psi_{i}\right\}_{i \in I}$.

Theorem 2.5. Let $\Xi=\left\{\Xi_{j}\right\}_{j \in I}, \Xi^{\prime}=\left\{\Xi_{j}^{\prime}\right\}_{j \in I}$ and $\Psi=\left\{\psi_{i}\right\}_{i \in I}, \Psi^{\prime}=\left\{\Psi_{i}^{\prime}\right\}_{i \in I}$ be g-orthonormal bases for $\mathcal{H}$ with respect to $\left\{\mathrm{W}_{\mathrm{j}}\right\}_{j \in \mathrm{I}}$ and $\left\{\mathrm{V}_{i}\right\}_{i \in \mathrm{I}}$ and let $\Lambda=\left\{\Lambda_{i}\right\}_{i \in \mathrm{I}}$ be a g-Bessel sequence for $\mathcal{H}$ with respect to $\left\{\mathrm{V}_{i}\right\}_{i \in \mathrm{I}}$. Denote the analysis matrix for $\Lambda$ with respect to $\Xi$ by $A$ and the $g$ - $R$-dual sequences of $\Lambda$ with respect to $(\Xi, \Psi)$ and $\left(\Xi^{\prime}, \Psi^{\prime}\right)$ by $\left\{\Gamma_{j}^{\wedge}\right\}_{j \in J},\left\{\Gamma_{j}^{\prime \wedge}\right\}_{j \in J}$, respectively. Then the following conditions are equivalent.

(i) $\Gamma_{j}^{\wedge}=\Gamma_{j}^{\prime \wedge}$ for all $j \in I$.

(ii) If $\mathrm{B}$ and $\mathrm{C}$ are the transition matrices from $\Xi$ to $\Xi^{\prime}$ and $\Psi$ to $\Psi^{\prime}$, respectively, then $\mathrm{AB}^{*}=\mathrm{CA}$.

Proof. Let $B=\left[B_{i j}\right]$ and $C=\left[C_{i j}\right]$. By the definition of $\left\{\Gamma_{j}^{\wedge}\right\}_{j \in J},\left\{\Gamma_{j}^{\prime \wedge}\right\}_{j \in J}$ for every $i, j \in I$ we have $\Psi_{i}\left(\Gamma_{j}^{\wedge}\right)^{*}=\Lambda_{i} \Xi_{j}^{*}$ and $\Psi_{i}^{\prime}\left(\Gamma_{j}^{\prime \wedge}\right)^{*}=\Lambda_{i} \Xi_{j}^{\prime *}$. Since

$$
\left[A B^{*}\right]_{i j}=\sum_{k \in I} A_{i k} B_{k j}^{*}=\sum_{k \in I} \Lambda_{i} \Xi_{k}^{*} \Xi_{k} \Xi_{j}^{\prime *}=\Lambda_{i}\left(\sum_{k \in I} \Xi_{k}^{*} \Xi_{k}\right) \Xi_{j}^{\prime *}=\Lambda_{i} \Xi_{j}^{\prime *}=\Psi_{i}^{\prime}\left(\Gamma_{j}^{\prime \wedge}\right)^{*}
$$

and

$$
[\mathrm{CA}]_{i j}=\sum_{k \in \mathrm{I}} C_{i k} A_{k j}=\sum_{k \in I} \Psi_{i}^{\prime} \Psi_{k}^{*} \Lambda_{k} \Xi_{j}^{*}=\sum_{k \in I} \Psi_{i}^{\prime} \Psi_{k}^{*} \Psi_{k}\left(\Gamma_{j}^{\wedge}\right)^{*}=\Psi_{i}^{\prime}\left(\sum_{k \in I} \Psi_{k}^{*} \Psi_{k}\right)\left(\Gamma_{j}^{\wedge}\right)^{*}=\Psi_{i}^{\prime}\left(\Gamma_{j}^{\wedge}\right)^{*}
$$

the conclusion follows.

Corollary 2.6. In addition to the hypothesis of Theorem 2.5 , if $\Lambda=\left\{\Lambda_{i}\right\}_{i \in \mathrm{I}}$ is a g-frame for $\mathcal{H}$ with respect to $\left\{\mathrm{V}_{i}\right\}_{\mathbf{i} \in \mathrm{I}}$ and $\left\{\Gamma_{j}^{\wedge}\right\}_{\mathbf{j} \in \mathrm{I}}=\left\{\Gamma_{\mathbf{j}}^{\prime \wedge}\right\}_{\mathbf{j} \in \mathrm{I}}$, then $\mathrm{A}^{*} \mathrm{C}^{*} \mathrm{AS} \mathrm{S}_{\Lambda}^{-1} \mathrm{~B}^{*}=\mathrm{I}$, where $\mathrm{I}$ is the identity matrix.

Proof. Let $\Lambda=\left\{\Lambda_{i}\right\}_{i \in I}$ be a g-frame for $\mathcal{H}$ with respect to $\left\{V_{i}\right\}_{i \in I}$. Definition 2.4 implies that $S_{\Lambda}^{-1} A^{*} A=I$. Thus, if $\Gamma_{j}^{\wedge}=\Gamma_{j}^{\prime \wedge}$ for all $j \in I$, then by Theorem 2.5, $A B^{*}=C A$. This implies $B^{*}=S_{\wedge}^{-1} A^{*} C A$. But B has to be unitary, which yields $A^{*} C^{*} A S_{\wedge}^{-1} B^{*}=I$.

Recall that two sequences $\left\{\Gamma_{j}\right\}_{j \in I}$ and $\left\{\Gamma_{j}^{\prime}\right\}_{j \in I}$ are called equivalent (unitarily equivalent) in $\mathcal{H}$ with respect to $\left\{W_{j}\right\}_{j \in I}$, if there exists a bounded linear invertible (unitary) operator $\mathrm{T}: \mathcal{H} \rightarrow \mathcal{H}$ such that $\mathrm{T} \Gamma_{j}^{*}=\Gamma_{j}^{*}$ for all $j \in \mathrm{I}$.

To have a better understanding of the different types of equivalency, we prove the following characterization result.

Theorem 2.7. In addition to the hypothesis of Theorem 2.5, if $\Gamma=\left\{\Gamma_{\mathbf{j}}^{\wedge}\right\}_{\mathbf{j} \in \mathrm{I}}$ and $\Gamma^{\prime}=\left\{\Gamma_{\mathbf{j}}^{\prime \wedge}\right\}_{\mathbf{j} \in \mathrm{I}}$ are g-frames for $\mathcal{H}$ with respect to $\left\{\mathrm{W}_{\mathrm{j}}\right\}_{j \in \mathrm{I}}$ and $\left\{\mathrm{V}_{\mathbf{j}}\right\}_{j \in \mathrm{I}}$, respectively, then the following statements hold.

(i) If $\Lambda=\left\{\Lambda_{i}\right\}_{i \in I}$ is a g-frame for $\mathcal{H}$ with respect to $\left\{\mathrm{V}_{i}\right\}_{\mathbf{i} \in \mathrm{I}}$, then $\left\{\Gamma_{j}^{\wedge}\right\}_{j \in \mathrm{I}}$ is equivalent to $\left\{\Gamma_{\mathbf{j}}^{\prime \wedge}\right\}_{j \in \mathrm{I}}$ in $\mathcal{H}$ with respect to $\left\{W_{j}\right\}_{j \in I}$ if and only if $\operatorname{ker}(A)=\operatorname{ker}\left(A B^{*}\right)$.

(ii) $\left\{\Gamma_{\mathbf{j}}^{\wedge}\right\}_{\mathbf{j} \in \mathrm{I}}$ is unitarily equivalent to $\left\{\Gamma_{\mathbf{j}}^{\prime \wedge}\right\}_{\mathbf{j} \in \mathrm{I}}$ in $\mathcal{H}$ with respect to $\left\{\mathrm{W}_{\mathbf{j}}\right\}_{\mathbf{j} \in \mathrm{I}}$, if and only if

$$
A^{*} A=\left(A B^{*}\right)^{*}\left(A B^{*}\right) .
$$

Moreover, if $\Lambda=\left\{\Lambda_{i}\right\}_{i \in I}$ is a g-frame for $\mathcal{H}$ with respect to $\left\{\bigvee_{i}\right\}_{i \in I}$, then the above is equivalent to $S_{\Lambda}=$ $\mathrm{BS}_{\wedge} \mathrm{B}^{*}$.

Proof.

(i) First we observe that, for every $g^{\prime}=\left\{g_{k}^{\prime}\right\}_{k \in I} \in\left(\sum_{j \in I} \oplus V_{j}\right)_{\ell^{2}}$ we have

$$
\sum_{k \in I}\left\|g_{k}^{\prime}\right\|^{2}=\sum_{k \in I}\left\langle g_{k}^{\prime}, g_{k}^{\prime}\right\rangle=\sum_{k \in I}\left\langle\sum_{i \in I} \Psi_{k}^{\prime} \Psi_{i}^{\prime *} g_{i}^{\prime}, g_{k}^{\prime}\right\rangle=\left\langle\sum_{i \in I}{\psi_{i}^{\prime}}_{i}^{\prime *} g_{i}^{\prime}, \sum_{k \in I} \Psi_{k}^{\prime *} g_{k}^{\prime}\right\rangle=\left\|\sum_{k \in I} \Psi_{k}^{\prime *} g_{k}^{\prime}\right\|^{2}
$$


Therefore,

$$
\sum_{k \in \mathrm{I}} \Psi_{k}^{\prime *} g_{k}^{\prime}=0 \Leftrightarrow g^{\prime}=0
$$

(Necessity). Suppose that $\left\{\Gamma_{j}^{\wedge}\right\}_{j \in I}$ is equivalent to $\left\{\Gamma_{j}^{\prime \wedge}\right\}_{j \in I}$ in $\mathcal{H}$ with respect to $\left\{W_{j}\right\}_{j \in I}$, then there exists a bounded linear invertible operator $\mathrm{T}: \mathcal{H} \rightarrow \mathcal{H}$ such that

$$
\mathrm{T}\left(\sum_{j \in \mathrm{I}}\left(\Gamma_{j}^{\wedge}\right)^{*} \mathrm{~g}_{j}\right)=\sum_{j \in \mathrm{I}}\left(\Gamma^{\prime \wedge}{ }_{j}^{\wedge}\right)^{*} g_{j}, \quad \forall\left\{g_{j}\right\}_{j \in I} \in\left(\sum_{j \in I} \oplus W_{j}\right)_{\ell^{2}}
$$

Now, $\mathrm{Ag}=0$ with $\mathrm{g}=\left\{\mathrm{g}_{\mathrm{j}}\right\}_{j \in \mathrm{I}}$, if and only if

$$
T^{-1}\left(\sum_{j \in I}\left(\Gamma_{j}^{\prime \wedge}\right)^{*} g_{j}\right)=\sum_{j \in I}\left(\Gamma_{j}^{\wedge}\right)^{*} g_{j}=\sum_{k \in I} \sum_{j \in I} \Psi_{k}^{*} \Lambda_{k} \Xi_{j}^{*} g_{j}=\sum_{k \in I} \sum_{j \in I} \Psi_{k}^{*} A_{k j} g_{j}=\sum_{k \in I} \Psi_{k}^{*}(A g)_{k}=0,
$$

if and only if

$$
\begin{aligned}
\sum_{k \in I} \Psi_{k}^{\prime *}\left(A B^{*} g\right)_{k} & =\sum_{k \in I} \Psi_{k}^{\prime *}\left(\sum_{j \in I}\left[A B^{*}\right]_{k j} g_{j}\right) \\
& =\sum_{k \in I} \sum_{j \in I} \sum_{i \in I} \Psi_{k}^{\prime *} A_{k i} B_{i j}^{*} g_{j} \\
& =\sum_{k \in I} \sum_{j \in I} \sum_{i \in I} \Psi_{k}^{\prime *} \Lambda_{k} \Xi_{i}^{*} \Xi_{i} \Xi_{j}^{*} g_{j} \\
& =\sum_{k \in I} \sum_{j \in I} \Psi_{k}^{\prime *} \Lambda_{k}\left(\sum_{i \in I} \Xi_{i}^{*} \Xi_{i} \Xi_{j}^{\prime *} g_{j}\right) \\
& =\sum_{k \in I} \sum_{j \in I} \Psi_{k}^{\prime *} \Lambda_{k} \Xi_{j}^{\prime *} g_{j}=\sum_{j \in I}\left(\Gamma_{j}^{\prime \wedge}\right)^{*} g_{j}=T^{-1}\left(\sum_{j \in I}\left(\Gamma_{j^{\prime}}^{\wedge}\right)^{*} g_{j}\right)=0,
\end{aligned}
$$

if and only if $A B^{*} g=0$.

(Sufficiency). Suppose that $\operatorname{ker}(A)=\operatorname{ker}\left(A B^{*}\right)$. Define the operator $\mathrm{T}$ as follows:

$$
\mathrm{T}: \operatorname{span}\left\{\left(\Gamma_{j}^{\wedge}\right)^{*}\left(W_{j}\right)\right\}_{j \in I} \rightarrow \operatorname{span}\left\{\left(\Gamma_{j}^{\prime \wedge}\right)^{*}\left(W_{j}\right)\right\}_{j \in I^{\prime}} \quad T\left(\sum_{j \in J}\left(\Gamma_{j}^{\wedge}\right)^{*} g_{j}\right)=\sum_{j \in J}\left(\Gamma_{j}^{\prime \wedge}\right)^{*} g_{j},
$$

for all $\mathrm{J} \subset \mathrm{I}$ with $|\mathrm{J}|<\infty$ and $\mathrm{g}_{j} \in W_{j}(j \in J)$. Let $\mathrm{C}, \mathrm{D}>0$ be the g-frame bounds for g-frame $\Lambda=\left\{\Lambda_{i}\right\}_{i \in I}$. Then we have

$$
\begin{aligned}
\left\|\mathrm{T}\left(\sum_{j \in J}\left(\Gamma_{j}^{\wedge}\right)^{*} g_{j}\right)\right\|^{2} & =\left\|\sum_{j \in J}\left(\Gamma_{j}^{\prime \wedge}\right)^{*} g_{j}\right\|^{2}=\left\|\sum_{k \in I} \sum_{j \in J} \Psi_{k}^{\prime *} \Lambda_{k} \Xi^{\prime *} g_{j}\right\|^{2} \\
& =\left\|\sum_{k \in I} \Psi^{\prime *} \Lambda_{k}\left(\sum_{j \in J} \Xi_{j}^{\prime *} g_{j}\right)\right\|^{2}=\sum_{k \in I}\left\|\Lambda_{k}\left(\sum_{j \in J} \Xi_{j}^{\prime *} g_{j}\right)\right\|^{2} \\
& \leqslant D\left\|\sum_{j \in J} \Xi_{j}^{\prime *} g_{j}\right\|^{2}=D \sum_{j \in J}\left\|g_{j}\right\|^{2}=D\left\|\sum_{j \in J} \Xi_{j}^{*} g_{j}\right\|^{2} \\
& \leqslant \frac{D}{C} \sum_{k \in I}\left\|\Lambda_{k}\left(\sum_{j \in J} \Xi_{j}^{*} g_{j}\right)\right\|^{2}=\frac{D}{C}\left\|\sum_{k \in I} \Psi_{k}^{*} \Lambda_{k}\left(\sum_{j \in J} \Xi_{j}^{*} g_{j}\right)\right\|^{2} \\
& =\frac{D}{C}\left\|\sum_{j \in J}\left(\sum_{k \in I} \Xi_{j} \Lambda_{k}^{*} \Psi_{k}\right)^{*} g_{j}\right\|^{2}=\frac{D}{C}\left\|\sum_{j \in J}\left(\Gamma_{j}^{\wedge}\right)^{*} g_{j}\right\|^{2} .
\end{aligned}
$$

This shows that $\mathrm{T}$ is a bounded linear operator. To prove invertibility of $\mathrm{T}$ we compute

$$
\begin{aligned}
\mathrm{T}\left(\sum_{j \in J}\left(\Gamma_{j}^{\wedge}\right)^{*} g_{j}\right) & =\sum_{j \in J}\left(\Gamma_{j}^{\prime \wedge}\right)^{*} g_{j}=\sum_{k \in I} \sum_{j \in J} \Psi_{k}^{\prime *} \Lambda_{k} \Xi_{j}^{\prime *} g_{j}=\sum_{k \in I} \sum_{j \in J} \Psi_{k}^{\prime *} \Lambda_{k}\left(\sum_{i \in I} \Xi_{i}^{*} \Xi_{i} \Xi_{j}^{\prime *} g_{j}\right) \\
& =\sum_{k \in I} \Psi_{k}^{\prime *}\left(\sum_{j \in J}\left[A B^{*}\right]_{k j} g_{j}\right)=\sum_{k \in I} \Psi_{k}^{\prime *}\left(A B^{*} g\right)_{k} .
\end{aligned}
$$


We also have

$$
\sum_{j \in J}\left(\Gamma_{j}^{\wedge}\right)^{*} g_{j}=\sum_{k \in I} \sum_{j \in J} \Psi_{k}^{*} \Lambda_{k} \Xi_{j}^{*} g_{j}=\sum_{k \in I} \Psi_{k}^{*}(A g)_{k}
$$

Hence,

$$
\mathrm{T}\left(\sum_{j \in J}\left(\Gamma_{j}^{\wedge}\right)^{*} g_{j}\right)=0 \Leftrightarrow \sum_{j \in J}\left(\Gamma_{j}^{\wedge}\right)^{*} g_{j}=0 .
$$

This implies that $T$ is invertible operator. Now, the g-completeness of $\Gamma$ and $\Gamma^{\prime}$ for $\mathcal{H}$ with respect to $\left\{W_{i}\right\}_{i \in I}$ implies that $T$ has an extension invertible on $\mathcal{H}$ and $T\left(\Gamma_{j}^{\wedge}\right)^{*}=\left(\Gamma_{j}^{\prime}{ }_{j}\right)^{*}$ for all $j \in I$.

(ii) First, we prove $\left[A^{*} A\right]_{i j}=\Gamma_{i}^{\wedge}\left(\Gamma_{j}^{\wedge}\right)^{*}$ and $\left[\left(A B^{*}\right)^{*}\left(A B^{*}\right)\right]_{i j}=\Gamma_{i}^{\prime \wedge}\left(\Gamma_{j}^{\prime \wedge}\right)^{*}$. To see this, we have

$$
\begin{aligned}
\Gamma_{i}^{\wedge}\left(\Gamma_{j}^{\wedge}\right)^{*} & =\left(\sum_{k \in I} \Xi_{i} \Lambda_{k}^{*} \Psi_{k}\right)\left(\sum_{m \in I} \Psi_{m}^{*} \Lambda_{m} \Xi_{j}^{*}\right)=\sum_{k \in I} \sum_{m \in I} \delta_{k m} \Xi_{i} \Lambda_{k}^{*} \Lambda_{m} \Xi_{j}^{*}=\sum_{k \in I} \Xi_{i} \Lambda_{k}^{*} \Lambda_{k} \Xi_{j}^{*} \\
& =\sum_{k \in I} A_{i k}^{*} A_{k j}=\left[A^{*} A\right]_{i j} .
\end{aligned}
$$

Moreover, we obtain

$$
\begin{aligned}
\Gamma_{i}^{\prime \wedge}\left(\Gamma_{j}^{\prime \wedge}\right)^{*} & =\left(\sum_{k \in I} \Xi_{i}^{\prime} \Lambda_{k}^{*} \Psi_{k}^{\prime}\right)\left(\sum_{m \in I} \Psi_{m}^{\prime *} \Lambda_{m} \Xi_{j}^{\prime *}\right) \\
& =\sum_{k \in I} \sum_{m \in I} \delta_{k m} \Xi_{i}^{\prime} \Lambda_{k}^{*} \Lambda_{m} \Xi_{j}^{\prime *}=\sum_{k \in I}\left(\Lambda_{k} \Xi_{i}^{\prime *}\right)^{*}\left(\Lambda_{k} \Xi_{j}^{\prime *}\right) \\
& =\sum_{k \in I}\left(\sum_{n \in I} \Lambda_{k} \Xi_{n}^{*} \Xi_{n} \Xi_{i}^{\prime *}\right)^{*}\left(\sum_{m \in I} \Lambda_{k} \Xi_{m}^{*} \Xi_{m} \Xi_{j}^{\prime *}\right) \\
& =\sum_{k \in I}\left(\sum_{n \in I} A_{k n} B_{n i}^{*}\right)^{*}\left(\sum_{m \in I} A_{k m} B_{m j}^{*}\right) \\
& =\sum_{k \in I}\left(A B^{*}\right)_{i k}^{*}\left(A B^{*}\right)_{k j}=\left[\left(A B^{*}\right)^{*}\left(A B^{*}\right)\right]_{i j} .
\end{aligned}
$$

Now, let $A^{*} A=\left(A B^{*}\right)^{*}\left(A B^{*}\right)$. Define the operator $T$ as follows:

$$
\mathrm{T}: \operatorname{span}\left\{\left(\Gamma_{j}^{\wedge}\right)^{*}\left(W_{j}\right)\right\}_{j \in I} \rightarrow \operatorname{span}\left\{\left(\Gamma_{j}^{\prime \wedge}\right)^{*}\left(W_{j}\right)\right\}_{j \in I^{\prime}} \quad T\left(\sum_{j \in J}\left(\Gamma_{j}^{\wedge}\right)^{*} g_{j}\right)=\sum_{j \in J}\left(\Gamma_{j}^{\prime \wedge}\right)^{*} g_{j},
$$

for all finite subsets $J \subset I$ and $g_{j} \in W_{j}(j \in J)$. Let $f_{1}, f_{2} \in \operatorname{span}\left\{\left(\Gamma_{j}^{\wedge}\right)^{*}\left(W_{j}\right)\right\}_{j \in I}$ as $f_{1}=\sum_{j \in J_{1}}\left(\Gamma_{j}^{\wedge}\right)^{*} g_{1 j}$ and $f_{2}=\sum_{j \in J_{2}}\left(\Gamma_{j}^{\wedge}\right)^{*} g_{2 j}$, we have

$$
\begin{aligned}
\left\langle\mathrm{Tf}_{1}, \mathrm{Tf}_{2}\right\rangle & =\left\langle\sum_{j \in J_{1}}\left(\Gamma_{j}^{\prime \wedge}\right)^{*} g_{1 j}, \sum_{k \in J_{2}}\left(\Gamma_{k}^{\prime \wedge}\right)^{*} g_{2 k}\right\rangle \\
& =\sum_{j \in J_{1}} \sum_{k \in J_{2}}\left\langle\Gamma_{k}^{\prime \wedge}\left(\Gamma_{j}^{\prime \wedge}\right)^{*} g_{1 j}, g_{2 k}\right\rangle \\
& =\left\langle\sum_{j \in J_{1}}\left(\Gamma_{j}^{\wedge}\right)^{*} g_{1 j}, \sum_{k \in J_{2}}\left(\Gamma_{k}^{\wedge}\right)^{*} g_{2 k}\right\rangle \\
& =\left\langle f_{1}, f_{2}\right\rangle .
\end{aligned}
$$

This implies that $T$ is a bounded linear surjective isometry operator. Thus, the g-completeness of $\Gamma$ and $\Gamma^{\prime}$ for $\mathcal{H}$ with respect to $\left\{W_{i}\right\}_{i \in I}$ implies that $T$ has an extension isometry on $\mathcal{H}$ and $T\left(\Gamma_{j}^{\wedge}\right)^{*}=\left(\Gamma_{j}^{\prime}{ }_{j}\right)^{*}$ for all $j \in I$. This shows that $\Gamma$ is unitarily equivalent to $\Gamma^{\prime}$ in $\mathcal{H}$ with respect to $\left\{W_{j}\right\}_{j \in I}$. The converse implication is obvious. Finally, if $\Lambda=\left\{\Lambda_{i}\right\}_{i \in I}$ is a g-frame for $\mathcal{H}$ with respect to $\left\{V_{i}\right\}_{i \in I}$, then, since $A^{*} A=S_{\Lambda}$, thus

$$
S_{\wedge}=A^{*} A=\left(A B^{*}\right)^{*}\left(A B^{*}\right)=B A^{*} A B^{*}=B S_{\wedge} B^{*} .
$$




\section{Characterizations of equivalence of the g-R-dual sequence}

In this section we first characterize all sequences with lower g-frame bound. Next, we obtain the gframe conditions for a sequence of operators and its $\mathrm{g}$-R-dual sequence. We also characterize those pairs of $g$-frames and their $\mathrm{g}$-R-dual sequences, which are equivalent (unitarily equivalent).

Recall that a family $\left\{\Lambda_{i}\right\}_{i \in I}$ is a g-frame sequence with respect to $\left\{V_{i}\right\}_{i \in I}$, if it is a $g$-frame for $\overline{\operatorname{span}}\left\{\Lambda_{i}^{*}\left(V_{i}\right)\right\}_{i \in I}$ with respect to $\left\{V_{i}\right\}_{i \in I}$.

There exists a characterization of frames which keeps the information about the frame bounds ([5, Lemma 5.5.5]). A similar result holds in g-frame situation.

Proposition 3.1. Let $\Lambda=\left\{\Lambda_{i} \in B\left(\mathcal{H}, V_{i}\right): i \in I\right\}$. Then the following conditions are equivalent.

(i) $\Lambda=\left\{\Lambda_{i}\right\}_{i \in I}$ is a $g$-frame sequence with respect to $\left\{V_{i}\right\}_{i \in I}$ with $g$-frame bounds $A$ and $B$.

(ii) The synthesis operator $\mathrm{T}_{\Lambda}^{*}$ is well-defined on $\left(\sum_{i \in \mathrm{I}} \oplus \mathrm{V}_{i}\right)_{\ell^{2}}$ such that:

$$
A\left\|g^{\prime}\right\|_{\ell^{2}}^{2} \leqslant\left\|T_{\Lambda}^{*} g^{\prime}\right\|^{2} \leqslant B\left\|g^{\prime}\right\|_{\ell^{2}}^{2}, \quad \forall g^{\prime} \in\left(\operatorname{ker}_{T_{\Lambda}^{*}}\right)^{\perp} .
$$

Proof. This follows immediately from [5, Lemma 5.5.5].

The next result shows a basic connection between a sequence of operators and its g-R-dual sequence which will be used frequently in what follows.

Theorem 3.2. Let $\Lambda=\left\{\Lambda_{i}\right\}_{i \in I}$ be a g-Bessel sequence for $\mathcal{H}$ with respect to $\left\{\bigvee_{i}\right\}_{i \in I}$. Then for every $\left\{g_{j}\right\}_{j \in I} \in$ $\left(\sum_{j \in I} \oplus W_{j}\right)_{\ell^{2}},\left\{g_{i}^{\prime}\right\}_{i \in I} \in\left(\sum_{i \in I} \oplus V_{i}\right)_{\ell^{2}}$ satisfying $f=\sum_{j \in I} \Xi_{j}^{*} g_{j}$ and $h=\sum_{i \in I} \Psi_{i}^{*} g_{i^{\prime}}^{\prime}$ we have

$$
\left\|\sum_{j \in I}\left(\Gamma_{j}^{\wedge}\right)^{*} g_{j}\right\|^{2}=\sum_{i \in I}\left\|\Lambda_{i} f\right\|^{2} \quad \text { and } \quad\left\|\sum_{i \in I} \Lambda_{i}^{*} g_{i}^{\prime}\right\|^{2}=\sum_{j \in I}\left\|\Gamma_{j}^{\wedge} h\right\|^{2} \text {. }
$$

Proof. It is easy to check that

$$
\begin{aligned}
\left\|\sum_{j \in I}\left(\Gamma_{j}^{\wedge}\right)^{*} g_{j}\right\|^{2} & =\left\|\sum_{j \in I}\left(\sum_{i \in I} \Xi_{j} \Lambda_{i}^{*} \Psi_{i}\right)^{*} g_{j}\right\|^{2}=\left\|\sum_{i \in I} \Psi_{i}^{*} \Lambda_{i} f\right\|^{2}=\left\langle\sum_{i \in I} \Psi_{i}^{*} \Lambda_{i} f, \sum_{j \in I} \Psi_{j}^{*} \Lambda_{j} f\right\rangle \\
& =\sum_{i \in I} \sum_{j \in I}\left\langle\Lambda_{i} f, \Psi_{i} \Psi_{j}^{*} \Lambda_{j} f\right\rangle \\
& =\sum_{i \in I} \sum_{j \in I}\left\langle\Lambda_{i} f, \delta_{i j} \Lambda_{j} f\right\rangle=\sum_{i \in I}\left\|\Lambda_{i} f\right\|^{2} .
\end{aligned}
$$

Similarly, the second claim follows from Theorem 2.3.

Corollary 3.3. Let $\Lambda=\left\{\Lambda_{i}\right\}_{i \in I}$ be a g-Bessel sequence for $\mathcal{H}$ with respect to $\left\{\bigvee_{i}\right\}_{i \in I}$. Then

$$
\left\|T_{\Gamma^{\wedge}}^{*}\left([f]_{\Xi}\right)\right\|=\left\|T_{\wedge} f\right\|_{\ell^{2}}, \quad\left\|T_{\Lambda}^{*}\left([f]_{\Psi}\right)\right\|=\left\|T_{\Gamma^{\wedge}} f\right\|_{\ell^{2}}
$$

for every $f \in \mathcal{H}$.

Proof. This follows immediately from Theorem 3.2.

There exists an interesting relation between the synthesis operator of $\Lambda=\left\{\Lambda_{i}\right\}_{i \in I}$ and the span of $\left\{\left(\Gamma_{j}^{\wedge}\right)^{*}\left(\mathbf{W}_{\mathbf{j}}\right)\right\}_{j \in I}$, which will turn out to be very useful in the sequel.

Theorem 3.4. Let $\Lambda=\left\{\Lambda_{i}\right\}_{i \in I}$ be a g-Bessel sequence for $\mathcal{H}$ with respect to $\left\{\mathrm{V}_{\boldsymbol{i}}\right\}_{\boldsymbol{i} \in \mathrm{I}}$ with g-R-dual sequence $\left\{\Gamma_{\mathbf{j}}\right\}_{j} \in \mathrm{I}$ with respect to $(\Xi, \Psi)$. Then the following statements hold.

(i) $f \in\left(\overline{\operatorname{span}}\left\{\left(\Gamma_{j}^{\wedge}\right)^{*}\left(W_{j}\right)\right\}_{j \in I}\right)^{\perp}$ if and only if $[\mathrm{f}]_{\Psi} \in \operatorname{ker}^{*}{ }_{\wedge}^{*}$. 
(ii) $\mathrm{f} \in\left(\overline{\operatorname{span}}\left\{\Lambda_{\mathbf{j}}^{*}\left(V_{\mathbf{j}}\right)\right\}_{\mathbf{j} \in \mathrm{I}}\right)^{\perp}$ if and only if $[\mathrm{f}]_{\Xi} \in \operatorname{ker} \mathrm{T}_{\Gamma^{*}}^{*}$.

Proof. Let $f \in \mathcal{H}$. First for each $j \in J$ and $g_{j} \in W_{j}$ we observe that

$$
\left\langle f,\left(\Gamma_{j}^{\wedge}\right)^{*} g_{j}\right\rangle=\sum_{i \in J}\left\langle f, \Psi_{i}^{*} \Lambda_{i} \Xi_{j}^{*} g_{j}\right\rangle=\left\langle\sum_{i \in J} \Lambda_{i}^{*} \Psi_{i} f, \Xi_{j}^{*} g_{j}\right\rangle=\left\langle T_{\Lambda}^{*}([f] \Psi), \Xi_{j}^{*} g_{j}\right\rangle .
$$

Since $\Xi=\left\{\Xi_{j}\right\}_{j \in J}$ is a g-orthonormal basis for $\mathcal{H}$ with respect to $\left\{W_{j}\right\}_{j \in I},\left\langle T_{\Lambda}^{*}\left([f]_{\Psi}\right), \Xi_{j}^{*} g_{j}\right\rangle=0$ for all $j \in I$ and $g_{j} \in W_{j}$, if and only if $T_{\Lambda}^{*}\left([f]_{\Psi}\right)=0$. Thus, $f \in\left(\operatorname{span}\left\{\left(\Gamma_{j}^{\wedge}\right)^{*}\left(W_{j}\right)\right\}_{j \in I}\right)^{\perp}$ is equivalent to $[f]_{\Psi} \in \operatorname{ker} T_{\Lambda}^{*}$. Similarly, the second claim follows from Theorem 2.3.

Corollary 3.5. Let $\Lambda=\left\{\Lambda_{i}\right\}_{i \in I}$ be a g-Bessel sequence for $\mathcal{H}$ with respect to $\left\{\bigvee_{i}\right\}_{i \in I}$ with $g$-R-dual sequence $\left\{\Gamma_{j}^{\wedge}\right\}_{j \in \mathrm{I}}$ with respect to $(\Xi, \Psi)$. Then

$$
\operatorname{dim}\left(\overline{\operatorname{span}}\left\{\left(\Gamma_{j}^{\wedge}\right)^{*}\left(W_{j}\right)\right\}_{j \in I}\right)^{\perp}=\operatorname{dim} \operatorname{ker} T_{\Lambda}^{*} \quad \text { and } \quad \operatorname{dim}\left(\overline{\operatorname{span}}\left\{\Lambda_{j}^{*}\left(V_{j}\right)\right\}_{j \in I}\right)^{\perp}=\operatorname{dim} \operatorname{ker} T_{\Gamma^{\wedge}}^{*} .
$$

Proof. This follows immediately from Theorem 3.4.

The next result shows a kind of equilibrium between a sequence of operators and its R-dual sequence. It can be viewed as a general version of [4, Proposition 13].

Corollary 3.6. The following conditions are equivalent.

(i) $\Lambda=\left\{\Lambda_{i}\right\}_{i \in I}$ is a g-frame sequence with respect to $\left\{\mathrm{V}_{i}\right\}_{i \in \mathrm{I}}$ with $g$-frame bounds $\mathrm{A}, \mathrm{B}$.

(ii) $\left\{\Gamma_{j}^{\wedge}\right\}_{j \in \mathrm{I}}$ is a g-frame sequence with respect to $\left\{\mathrm{W}_{\mathbf{j}}\right\}_{\mathbf{j} \in \mathrm{I}}$ with $\mathrm{g}$-frame bounds $\mathrm{A}, \mathrm{B}$.

(iii) $\left\{\Gamma_{\mathbf{j}}^{\wedge}\right\}_{\mathbf{j} \in \mathrm{I}}$ is a g-Riesz basic sequence with respect to $\left\{\mathrm{W}_{\mathbf{j}}\right\}_{j \in \mathrm{I}}$ with g-frame bounds $\mathrm{A}, \mathrm{B}$.

Proof. (i) $\Leftrightarrow$ (ii). The Proposition 3.1 and Theorem 3.4 conclude that $\Lambda=\left\{\Lambda_{i}\right\}_{i \in I}$ is a g-frame sequence with respect to $\left\{V_{i}\right\}_{i \in I}$ with g-frame bounds $A, B$ if and only if

$$
A \|[f]]_{\Psi}\left\|_{\ell^{2}}^{2} \leqslant\right\| T_{\Lambda}^{*}([f] \Psi)\left\|^{2} \leqslant B\right\|[f]_{\Psi} \|_{\ell^{2}}^{2}
$$

for all $f \in \overline{\operatorname{span}}\left\{\left(\Gamma_{j}^{\wedge}\right)^{*}\left(W_{j}\right)\right\}_{j \in I}$. Now, Corollary 3.3 implies

$$
A\|f\|^{2} \leqslant\left\|T_{\Gamma^{\wedge}} f\right\|_{\ell^{2}}^{2} \leqslant B\|f\|^{2} .
$$

(i) $\Leftrightarrow$ (iii). This equivalence follows immediately from Theorem 3.2.

The dimension condition in Corollary 3.5 will play a crucial role for the g-R-dual sequence. Using Corollary 3.5 we can derive a simple characterization of a g-Riesz basic sequence being a g-R-dual sequence of a g-frame in the tight case.

Theorem 3.7. Let $\Lambda=\left\{\Lambda_{i}\right\}_{i \in \mathrm{I}}$ be a A-tight g-frame for $\mathcal{H}$ with respect to $\left\{\mathrm{V}_{i}\right\}_{i \in \mathrm{I}}$ and let $\left\{\Gamma_{j}\right\}_{j \in \mathrm{I}}$ be an A-tight $g$-Riesz basic sequence in $\mathcal{H}$ with respect to $\left\{\mathrm{W}_{\mathbf{j}}\right\}_{j \in \mathrm{I}}$. Then $\left\{\Gamma_{j}\right\}_{j \in \mathrm{I}}$ is a $g$-R-dual sequence of $\left\{\Lambda_{i}\right\}_{i \in \mathrm{I}}$ with respect to $(\Xi, \Psi)$, if and only if

$$
\operatorname{dim}\left(\overline{\operatorname{span}}\left\{\Gamma_{j}^{*}\left(W_{j}\right)\right\}_{j \in I}\right)^{\perp}=\operatorname{dim} \operatorname{ker} T_{\Lambda}^{*} .
$$

Proof. The necessity of the condition in (3.1) follows from Corollary 3.5. Now, assume that (3.1) holds. Then, according to Lemma 1.6 the sequence $\left\{\frac{1}{\sqrt{A}} \Gamma_{j}\right\}_{j \in I}$ is a g-orthonormal system for $\mathcal{H}$ with respect to $\left\{W_{j}\right\}_{j \in I}$. Suppose that $\Xi=\left\{\Xi_{j}\right\}_{j \in I}$ and $\Psi=\left\{\Psi_{i}\right\}_{i \in I}$ are g-orthonormal bases for $\mathcal{H}$ with respect to $\left\{W_{j}\right\}_{j \in I}$ and $\left\{V_{i}\right\}_{i \in I}$, respectively. Consider the g-R-dual $\left\{\Theta_{j}\right\}_{j \in I}$ of $\Lambda=\left\{\Lambda_{i}\right\}_{i \in I}$ with respect to $(\Xi, \Psi)$, i.e., $\Theta_{j}=\sum_{i \in I} \Xi_{j} \Lambda_{i}^{*} \Psi_{i}, j \in I$. By Corollary $3.6\left\{\Theta_{j}\right\}_{j \in I}$ is an A-tight g-Riesz basic sequence with respect to 
$\left\{W_{j}\right\}_{j \in I}$ and hence $\left\{\frac{1}{\sqrt{A}} \Theta_{j}\right\}_{j \in I}$ is also a g-orthonormal system for $\mathcal{H}$ with respect to $\left\{W_{j}\right\}_{j \in I}$. By Corollary 3.5 and (3.1),

$$
\operatorname{dim}\left(\overline{\operatorname{span}}\left\{\Theta_{j}^{*}\left(W_{j}\right)\right\}_{j \in I}\right)^{\perp}=\operatorname{dim} \operatorname{ker} T_{\Lambda}^{*}=\operatorname{dim}\left(\overline{\operatorname{span}}\left\{\Gamma_{j}^{*}\left(W_{j}\right)\right\}_{j \in I}\right)^{\perp} .
$$

In case $\left(\overline{\operatorname{span}}\left\{\Theta_{j}^{*}\left(W_{j}\right)\right\}_{j \in I}\right)^{\perp}=\left(\overline{\operatorname{span}}\left\{\Gamma_{j}^{*}\left(W_{j}\right)\right\}_{j} \in I\right)^{\perp}=\{0\}$, the g-orthonormality of the sequences $\left\{\frac{1}{\sqrt{A}} \Theta_{i}\right\}_{i \in I}$ and $\left\{\frac{1}{\sqrt{A}} \Gamma_{i}\right\}_{i \in I}$ implies that there exists unitary operator

$$
\mathrm{U}: \mathcal{H} \rightarrow \mathcal{H}, \quad \text { by } \quad \Gamma_{\mathrm{j}}=\Theta_{\mathrm{j}} \mathrm{U}^{*}, \forall \mathrm{j} \in \mathrm{I} .
$$

In case $\left(\overline{\operatorname{span}}\left\{\Theta_{j}^{*}\left(W_{j}\right)\right\}_{j \in I}\right)^{\perp} \neq\{0\}$, letting $\left\{\Phi_{j}\right\}_{j \in I}$ and $\left\{\Omega_{j}\right\}_{j \in I}$ be g-orthonormal bases for

$$
\left(\overline{\operatorname{span}}\left\{\Theta_{j}^{*}\left(W_{j}\right)\right\}_{j \in I}\right)^{\perp} \quad \text { and } \quad\left(\overline{\operatorname{span}}\left\{\Gamma_{j}^{*}\left(W_{j}\right)\right\}_{j \in I}\right)^{\perp},
$$

with respect to $\left\{W_{j}\right\}_{j \in I}$, respectively, (3.2) implies that there exists unitary operator

$$
\mathrm{U}: \mathcal{H} \rightarrow \mathcal{H}, \quad \text { by } \quad \Gamma_{\mathrm{j}}=\Theta_{\mathrm{j}} \mathrm{U}^{*}, \quad \Omega_{\mathrm{j}}=\Phi_{\mathrm{j}} \mathrm{U}^{*} \forall \mathrm{j} \in \mathrm{I} .
$$

In both cases, we have

$$
\Gamma_{j}=\Theta_{j} \mathrm{U}^{*}=\left(\sum_{i \in \mathrm{I}} \Xi_{j} \wedge_{i}^{*} \Psi_{i}\right) \mathrm{U}^{*}=\sum_{i \in \mathrm{I}} \Xi_{j} \Lambda_{i}^{*} \Psi_{i} \mathrm{U}^{*}, \forall j \in \mathrm{I},
$$

which shows that $\left\{\Gamma_{j}\right\}_{j \in I}$ is a g-R-dual sequence of $\left\{\Lambda_{i}\right\}_{i \in I}$ with respect to $\left\{\Xi_{j}\right\}_{j \in I}$ and $\left\{\Psi_{i} U^{*}\right\}_{i \in I}$.

The following result is about different types of equivalence of g-frames, which is taken from [12]. This result will moreover be employed in several proofs in the sequel.

Proposition 3.8. Let $\Lambda=\left\{\Lambda_{i}\right\}_{i \in \mathrm{I}}$ and $\Lambda^{\prime}=\left\{\Lambda_{i}^{\prime}\right\}_{i \in \mathrm{I}}$ be Parseval g-frames for $\mathcal{H}_{1}$ and $\mathcal{H}_{2}$ with respect to $\left\{\mathrm{V}_{i}\right\}_{i \in \mathrm{I}}$, respectively. Then $\Lambda$ is unitarily equivalent to $\Lambda^{\prime}$ if and only if the analysis operators $\mathrm{T}_{\Lambda}$ and $\mathrm{T}_{\Lambda^{\prime}}$ have the same range. Likewise, two g-frames with respect to $\left\{\mathrm{V}_{i}\right\}_{i \in \mathrm{I}}$ are equivalent if and only if their analysis operators have the same range.

In the following we characterize those pairs of g-frames and their g-R-dual sequences, which are equivalent (unitarily equivalent).

Theorem 3.9. Let $\left\{\Lambda_{i}\right\}_{i \in \mathrm{I}}$ and $\left\{\Lambda_{i}^{\prime}\right\}_{i \in \mathrm{I}}$ be g-frames for $\mathcal{H}$ with respect to $\left\{\mathrm{V}_{i}\right\}_{i \in \mathrm{I}}$. Then

(i) $\left\{\Lambda_{i}\right\}_{i \in \mathrm{I}}$ is equivalent to $\left\{\Lambda_{i}^{\prime}\right\}_{i \in \mathrm{I}}$ in $\mathcal{H}$ with respect to $\left\{\mathrm{V}_{i}\right\}_{i \in \mathrm{I}}$ if and only if

$$
\overline{\operatorname{span}}\left\{\left(\Gamma_{\mathbf{j}}^{\wedge}\right)^{*}\left(\mathrm{~W}_{\mathbf{j}}\right)\right\}_{\mathbf{j} \in \mathrm{I}}=\overline{\operatorname{span}}\left\{\left(\Gamma_{\mathbf{j}}^{\Lambda^{\prime}}\right)^{*}\left(\mathrm{~W}_{\mathbf{j}}\right)\right\}_{\mathbf{j} \in \mathrm{I}} ;
$$

(ii) $\left\{\Lambda_{i}\right\}_{i \in \mathrm{I}}$ is unitarily equivalent to $\left\{\Lambda_{i}^{\prime}\right\}_{i \in \mathrm{I}}$ in $\mathcal{H}$ with respect to $\left\{\mathrm{V}_{i}\right\}_{i \in \mathrm{I}}$ if and only if $S_{\Gamma^{\wedge}}=S_{\Gamma^{\prime}{ }^{\prime}}$;

(iii) $\left\{\Gamma_{j}^{\wedge}\right\}_{j \in \mathrm{I}}$ is unitarily equivalent to $\left\{\Gamma_{j}^{\Lambda^{\prime}}\right\}_{j \in \mathrm{I}}$ in $\mathcal{H}$ with respect to $\left\{\mathrm{W}_{\mathrm{j}}\right\}_{j \in \mathrm{I}}$ if and only if $\mathrm{S}_{\Lambda}=\mathrm{S}_{\Lambda^{\prime}}$.

Proof.

(i) By Proposition 3.8, $\left\{\Lambda_{i}\right\}_{i \in \mathrm{I}}$ and $\left\{\Lambda_{i}^{\prime}\right\}_{i \in \mathrm{I}}$ are equivalent in $\mathcal{H}$ with respect to $\left\{\mathrm{V}_{i}\right\}_{i \in \mathrm{I}}$, if and only if $\mathcal{R}_{\mathrm{T}_{\Lambda}}=$ $\mathcal{R}_{\mathrm{T}_{\Lambda^{\prime}}}$ and hence $\operatorname{ker} \mathrm{T}_{\Lambda}^{*}=\operatorname{ker} \mathrm{T}_{\Lambda^{\prime}}^{*}$. Now the claim follows from Theorem 3.4.

(ii) Using Propositions 3.1 and 3.8, $\left\{\Lambda_{i}\right\}_{i \in I}$ is unitarily equivalent to $\left\{\Lambda_{i}^{\prime}\right\}_{i \in I}$ if and only if

$$
\left\|\sum_{i \in I} \Lambda_{i}^{*} g_{i}^{\prime}\right\|^{2}=\left\|\sum_{i \in I} \Lambda_{i}^{\prime *} g_{i}^{\prime}\right\|^{2}, \quad \forall\left\{g_{i}^{\prime}\right\}_{i \in I} \in\left(\operatorname{ker} T_{\Lambda}^{*}\right)^{\perp}
$$

By Theorem 3.2, this in turn is equivalent to

$$
\left\langle S_{\Gamma^{\wedge}} f, f\right\rangle=\sum_{j \in I}\left\|\Gamma_{j}^{\wedge} f\right\|^{2}=\sum_{j \in I}\left\|\Gamma_{j}^{\wedge^{\prime}} f\right\|^{2}=\left\langle S_{\Gamma^{\wedge^{\prime}}} f, f\right\rangle,
$$

for all $f \in \mathcal{H}$ and $g_{i}^{\prime}=\Psi_{i} f(i \in I)$. It follows that $S_{\Gamma^{\wedge}}=S_{\Gamma^{\wedge^{\prime}}}$, as required. 
(iii) The proof follows immediately from (ii) and Theorem 2.3.

Corollary 3.10. Let $\left\{\Lambda_{i}\right\}_{i \in I}$ be a g-frame for $\mathcal{H}$ with respect to $\left\{\mathrm{V}_{i}\right\}_{i \in \mathrm{I}}$. Then

$$
\overline{\operatorname{span}}\left\{\left(\Gamma_{j}^{\wedge}\right)^{*}\left(W_{j}\right)\right\}_{j \in I}=\overline{\operatorname{span}}\left\{\left(\Gamma_{j}^{\hat{\Lambda}}\right)^{*}\left(W_{j}\right)\right\}_{j \in I},
$$

where $\left\{\widehat{\Lambda}_{i}\right\}_{i \in \mathrm{I}}$ is the canonical dual g-frame of $\left\{\Lambda_{i}\right\}_{i \in \mathrm{I}}$.

Proof. Since $\left\{\widehat{\Lambda}_{i}\right\}_{i \in I}$ is equivalent to $\left\{\Lambda_{i}\right\}_{i \in I}$, this claim follows from Theorem 3.9.

\section{Duality properties of the g-R-dual sequence}

In this section we characterize all properties of a g-Bessel sequence in terms of properties of their g-Rdual sequence. We will study properties of dual g-frames and canonical dual g-frames. This is a general version of duality principle for g-frames which follows from the Casazza duality relations [4].

The next result gives an explicit form for $\mathrm{g}$-R-dual sequence of the canonical dual g-frame.

Theorem 4.1. Let $\left\{\Lambda_{i}\right\}_{i \in I}$ and $\left\{\Omega_{i}\right\}_{i \in I}$ be g-frames for $\mathcal{H}$ with respect to $\left\{\bigvee_{i}\right\}_{i \in I}$. Then $\left\{\Omega_{i}\right\}_{i \in I}$ is a dual g-frame of $\left\{\Lambda_{i}\right\}_{i \in \mathrm{I}}$ if and only if $g$-R-dual sequences $\left\{\Gamma_{j}^{\Lambda}\right\}_{j \in \mathrm{I}}$ and $\left\{\Gamma_{j}^{\Omega}\right\}_{j \in \mathrm{I}}$ are g-biorthogonal, i.e.,

$$
\Gamma_{i}^{\wedge}\left(\Gamma_{j}^{\Omega}\right)^{*} g_{j}=\Gamma_{i}^{\Omega}\left(\Gamma_{j}^{\wedge}\right)^{*} g_{j}=\delta_{i j} g_{j}, \quad \forall i, j \in I, g_{j} \in W_{j} .
$$

Proof. Let $\left\{\Omega_{i}\right\}_{i \in I}$ be a dual g-frame of $\left\{\Lambda_{i}\right\}_{i \in I}$. By definition of $\left\{\Gamma_{j}^{\Omega}\right\}_{j \in I}$ and $\left\{\Gamma_{j}^{\wedge}\right\}_{j \in I}$ for every $i, j \in I$ and $g_{j} \in W_{j}$ we have

$$
\begin{aligned}
\Gamma_{i}^{\wedge}\left(\Gamma_{j}^{\Omega}\right)^{*} g_{j} & =\sum_{k \in I} \Xi_{i} \Lambda_{k}^{*} \Psi_{k}\left(\sum_{m \in I} \Xi_{j} \Omega_{m}^{*} \Psi_{m}\right)^{*} g_{j} \\
& =\sum_{k \in I} \sum_{m \in I} \Xi_{i} \Lambda_{k}^{*} \Psi_{k} \Psi_{m}^{*} \Omega_{m} \Xi_{j}^{*} g_{j} \\
& =\sum_{k \in I} \Xi_{i} \Lambda_{k}^{*} \Omega_{k} \Xi_{j}^{*} g_{j}=\Xi_{i}\left(\sum_{k \in I} \Lambda_{k}^{*} \Omega_{k} \Xi_{j}^{*} g_{j}\right)=\Xi_{i} \Xi_{j}^{*} g_{j}=\delta_{i j} g_{j} .
\end{aligned}
$$

The converse implication similarly follows from Theorem 2.3.

Corollary 4.2. Let $\Lambda=\left\{\Lambda_{i}\right\}_{i \in I}$ be a g-frame for $\mathcal{H}$ with respect to $\left\{\bigvee_{i}\right\}_{i \in I}$ with canonical dual g-frame denoted by $\left\{\widehat{\Lambda}_{i}\right\}_{i \in \mathrm{I}}$. Then the $g$-R-dual sequences $\left\{\Gamma_{j}^{\wedge}\right\}_{j \in \mathrm{I}}$ and $\left\{\Gamma_{j} \hat{\Lambda}_{j \in \mathrm{I}}\right.$ are g-biorthogonal, i.e.,

$$
\Gamma_{i}^{\wedge}\left(\Gamma_{j}^{\hat{\Lambda}}\right)^{*} g_{j}=\Gamma_{i}^{\hat{\wedge}}\left(\Gamma_{j}^{\wedge}\right)^{*} g_{j}=\delta_{i j} g_{j}
$$

for all $i, j \in I$ and $g_{j} \in W_{j}$. Thus $\left\{\Gamma_{j}^{\hat{\Lambda}}\right\}_{j \in I}$ is the dual g-Riesz basic sequence of $\left\{\Gamma_{j}^{\wedge}\right\}_{j \in I}$.

The next result is a characterization of tight g-frames in terms of their $\mathrm{g}$-R-dual sequence.

Corollary 4.3. $\left\{\Lambda_{i}\right\}_{i \in I}$ is an A-tight g-frame for $\mathcal{H}$ with respect to $\left\{\mathrm{V}_{i}\right\}_{i \in \mathrm{I}}$ if and only if $g$-R-dual sequence $\left\{\frac{1}{\sqrt{A}} \Gamma_{j}^{\Lambda}\right\}_{j \in I}$ is a g-orthonormal system for $\mathcal{H}$ with respect to $\left\{\mathrm{W}_{\mathbf{j}}\right\}_{\mathfrak{j} \in \mathrm{I}}$. Thus the sequence $\left\{\Lambda_{i}\right\}_{\mathfrak{i} \in \mathrm{I}}$ is a Parseval $g$-frame if and only if, its $g$-R-dual sequence is an orthonormal system.

Proof. This follows immediately from Lemma 1.6, Corollary 3.6, and Theorem 4.2.

Theorem 4.4. Let $\left\{\Lambda_{i}\right\}_{i \in \mathrm{I}}$ and $\left\{\Omega_{i}\right\}_{i \in \mathrm{I}}$ be g-frames for $\mathcal{H}$ with respect to $\left\{\mathrm{V}_{i}\right\}_{i \in \mathrm{I}}$. Then $\left\{\Omega_{i}\right\}_{i \in \mathrm{I}}$ is a dual g-frame of $\left\{\Lambda_{i}\right\}_{i \in I}$ if and only if, there exists a g-Bessel sequence $\left\{\Theta_{j}\right\}_{j \in I}$ for $\left(\overline{\operatorname{span}}\left\{\left(\Gamma_{j}^{\wedge}\right)^{*}\left(W_{j}\right)\right\}_{j \in I}\right)^{\perp}$ with respect to $\left\{W_{j}\right\}_{j \in I}$, such that $\Gamma_{\mathfrak{j}}^{\Omega}=\Gamma_{j}^{\hat{\Lambda}}+\Theta_{j}$ for all $j \in I$. 
Proof. Suppose that $\left\{\Omega_{i}\right\}_{i \in I}$ is a dual g-frame of $\left\{\Lambda_{i}\right\}_{i \in I}$. By Theorem 4.1 we have

$$
\begin{aligned}
\left\langle\left(\Gamma_{i}^{\Omega}-\Gamma_{i}^{\hat{\Lambda}}\right)^{*} g_{i},\left(\Gamma_{j}^{\wedge}\right)^{*} g_{j}\right\rangle & =\left\langle g_{i},\left(\Gamma_{i}^{\Omega}-\Gamma_{i}^{\hat{\Lambda}}\right)\left(\Gamma_{j}^{\wedge}\right)^{*} g_{j}\right\rangle=\left\langle g_{i}, \Gamma_{i}^{\Omega}\left(\Gamma_{j}^{\wedge}\right)^{*} g_{j}\right\rangle-\left\langle g_{i}, \Gamma_{i}^{\hat{\Lambda}}\left(\Gamma_{j}^{\wedge}\right)^{*} g_{j}\right\rangle \\
& =\left\langle g_{i}, \delta_{i j} g_{j}\right\rangle-\left\langle g_{i}, \delta_{i j} g_{j}\right\rangle=0,
\end{aligned}
$$

for all $i, j \in I$ and $g_{i} \in W_{i}, g_{j} \in W_{j}$. Thus, Definition 2.1 implies that $\Theta_{j}=\Gamma_{j}^{\Omega}-\Gamma_{j}^{\hat{\Lambda}}$ is a g-Bessel sequence for $\left(\overline{\operatorname{span}}\left\{\left(\Gamma_{j}^{\wedge}\right)^{*}\left(W_{j}\right)\right\}_{j \in I}\right)^{\perp}$ with respect to $\left\{W_{j}\right\}_{j \in I}$ and $\Gamma_{j}^{\Omega}=\Gamma_{j}^{\hat{\Lambda}}+\Theta_{j}$. Now for the opposite implication, suppose that there exists a g-Bessel sequence $\left\{\Theta_{j}\right\}_{j \in I}$ for $\left(\overline{\operatorname{span}}\left\{\left(\Gamma_{j}^{\wedge}\right)^{*}\left(W_{j}\right)\right\}_{j \in I}\right)^{\perp}$ with respect to $\left\{W_{j}\right\}_{j \in I}$, such that $\Gamma_{j}^{\Omega}=\Gamma_{j}^{\hat{\Lambda}}+\Theta_{j}$ for all $j \in$ I. By Theorem 2.3, we have

$$
\Omega_{i}=\widehat{\Lambda}_{i}+\sum_{j \in I} \Psi_{i}\left(\Theta_{j}\right)^{*} \Xi_{j} \quad \text { for all } i \in I
$$

So, for each $f \in \mathcal{H}$

$$
\sum_{i \in I} \Lambda_{i}^{*} \Omega_{i} f=\sum_{i \in I} \Lambda_{i}^{*}\left(\widehat{\Lambda}_{i}+\sum_{j \in I} \Psi_{i} \Theta_{j}^{*} \Xi_{j}\right) f=\sum_{i \in I} \Lambda_{i}^{*} \widehat{\Lambda}_{i} f+\sum_{i \in I} \sum_{j \in I} \Lambda_{i}^{*} \Psi_{i} \Theta_{j}^{*} \Xi_{j} f=f+\sum_{j \in I} \sum_{i \in I} \Lambda_{i}^{*} \Psi_{i} \Theta_{j}^{*} \Xi_{j} f,
$$

since $\Theta_{j}^{*} \Xi_{j} f \in\left(\overline{\operatorname{span}}\left\{\left(\Gamma_{j}^{\wedge}\right)^{*}\left(W_{j}\right)\right\}_{j \in I}\right)^{\perp}$ for all $j \in$ I. Theorem 3.4 implies that

$$
\sum_{i \in \mathrm{I}} \Lambda_{i}^{*} \Psi_{i} \Theta_{j}^{*} \Xi_{j} f=0
$$

This proves that $\left\{\Omega_{i}\right\}_{i \in I}$ is a dual g-frame of $\left\{\Lambda_{i}\right\}_{i \in I}$.

Among the dual g-frames the canonical dual g-frame is distinguished by the following properties.

Theorem 4.5. Let $\Lambda=\left\{\Lambda_{i}\right\}_{i \in I}$ be a g-frame for $\mathcal{H}$ with respect to $\left\{\bigvee_{i}\right\}_{i \in I}$ with canonical dual g-frame denoted by $\left\{\widehat{\Lambda}_{i}\right\}_{i \in \mathrm{I}}$ and let $\left\{\Omega_{i}\right\}_{i \in \mathrm{I}}$ be a dual g-frame of $\left\{\Lambda_{i}\right\}_{i \in \mathrm{I}}$. Then

$$
\left\|\Gamma_{j}^{\widehat{\Lambda}}\right\| \leqslant\left\|\Gamma_{j}^{\Omega}\right\| \text { for all } j \in I,
$$

with equality if and only if $\left\{\Omega_{j}\right\}_{j \in I}=\left\{\widehat{\Lambda}_{j}\right\}_{j \in I}$.

Proof. By Theorem 4.4, $\left\{\Omega_{i}\right\}_{i \in I}$ is a dual g-frame of $\left\{\Lambda_{i}\right\}_{i \in I}$ if and only if $\Gamma_{j}^{\Omega}=\Gamma_{j}^{\hat{\Lambda}}+\Theta_{j}$, where $\left(\Gamma_{j}^{\hat{\Lambda}}\right)^{*} g \in$ $\overline{\operatorname{span}}\left\{\left(\Gamma_{j}^{\wedge}\right)^{*}\left(W_{j}\right)\right\}_{j \in I}$ and $\Theta_{j}^{*} g \in\left(\overline{\operatorname{span}}\left\{\left(\Gamma_{j}^{\wedge}\right)^{*}\left(W_{j}\right)\right\}_{j \in I}\right)^{\perp}$ for all $j \in I, g \in W_{j}$. Hence

$$
\begin{aligned}
\left\|\Gamma_{j}^{\Omega}\right\|^{2}=\left\|\left(\Gamma_{j}^{\Omega}\right)^{*}\right\|^{2} & =\sup _{\|g\|=1}\left\|\left(\Gamma_{j}^{\Omega}\right)^{*} g\right\|^{2}=\sup _{\|g\|=1}\left\|\left(\Gamma_{j}^{\hat{\Lambda}}\right)^{*} g\right\|^{2}+\sup _{\|g\|=1}\left\|\Theta_{j}^{*} g\right\|^{2} \\
& =\left\|\left(\Gamma_{j}^{\hat{\Lambda}}\right)^{*}\right\|^{2}+\left\|\Theta_{j}^{*}\right\|^{2}=\left\|\Gamma_{j}^{\widehat{\Lambda}}\right\|^{2}+\left\|\Theta_{j}\right\|^{2} \geqslant\left\|\Gamma_{j}^{\widehat{\Lambda}}\right\|^{2},
\end{aligned}
$$

with equality if and only if $\left\{\Omega_{j}\right\}_{j \in I}=\left\{\widehat{\Lambda}_{\mathbf{j}}\right\}_{j \in I}$.

\section{Acknowledgment}

The authors would like to thank the anonymous reviewers for carefully reading of the manuscript and giving useful comments, which will help to improve the paper. The authors were supported by Islamic Azad University, Central Tehran Branch. 


\section{References}

[1] M. S. Asgari, Operator-valued bases on Hilbert spaces, J. Linear Topol. Algebr., 2 (2013), 201-218. 1, 1

[2] M. S. Asgari, H. R. Rahimi, Generalized frames for operators in Hilbert spaces, Infin. Dimens. Anal. Quantum Probab. Relat. Top., 17 (2014), 20 pages. 1, 1

[3] P. G. Casazza, G. Kutyniok, Frames of subspaces, Wavelets, frames and operator theory, Contemp. Math., Amer. Math. Soc., Providence, RI, 345 (2004), 87-113. 1

[4] P. G. Casazza, G. Kutyniok, M. C. Lammers, Duality principles in frame theory, J. Fourier Anal. Appl., 10 (2004), 383-408. 1, 2, 3, 4

[5] O. Christensen, An introduction to frames and Riesz bases, Second edition, Applied and Numerical Harmonic Analysis, Birkhäuser/Springer, [Cham], (2016). 1, 3, 3

[6] O. Christensen, H. O. Kim, R. Y. Kim, On the duality principle by Casazza, Kutyniok, and Lammers, J. Fourier Anal. Appl., 17 (2011), 640-655. 1

[7] O. Christensen, X. C. Xiao, Y. C. Zhu, Characterizing R-duality in Banach spaces, Acta Math. Sin. (Engl. Ser.), 29 (2013), 75-84. 1

[8] I. Daubechies, A. Grossmann, Y. Meyer, Painless nonorthogonal expansions, J. Math. Phys., 27 (1986), 1271-1283. 1

[9] R. J. Duffin, A. C. Schaeffer, A class of nonharmonic Fourier series, Trans. Amer. Math. Soc., 72 (1952), 341-366. 1

[10] X.-X. Guo, g-bases in Hilbert spaces, Abstr. Appl. Anal., 2012 (2012), 14 pages. 1

[11] X.-X. Guo, Operator parameterizations of g-frames, Taiwanese J. Math., 18 (2014), 313-328. 1

[12] A. Najati, A. Rahimi, Generalized frames in Hilbert spaces, Bull. Iranian Math. Soc., 35 (2009), 97-109. 3

[13] A. Ron, Z.-W. Shen, Weyl-Heisenberg frames and Riesz bases in $\mathrm{L}_{2}\left(\mathbf{R}^{\mathrm{d}}\right)$, Duke Math. J., 89 (1997), 237-282. 1

[14] D. T. Stoeva, O. Christensen, On R-duals and the duality principle in Gabor analysis, J. Fourier Anal. Appl., 21 (2015), 383-400. 1

[15] W.-C. Sun, G-frames and g-Riesz bases, J. Math. Anal. Appl., 322 (2006), 437-452. 1

[16] W.-C. Sun, Stability of g-frames, J. Math. Anal. Appl., 326 (2007), 858-868. 1

[17] J. Wexler, S. Raz, Discrete gabor expansions, Signal Process., 21 (1990), 207-221. 1

[18] X. M. Xian, Y. C. Zhu, Duality principles of frames in Banach spaces, (Chinese) Acta Math. Sci. Ser. A Chin. Ed., 29 (2009), 94-102. 1 